\title{
Hepatic insulin signaling regulates VLDL secretion and atherogenesis in mice
}

\author{
Seongah Han, Chien-Ping Liang, Marit Westerterp, Takafumi Senokuchi, Carrie L. Welch, \\ Qizhi Wang, Michihiro Matsumoto, Domenico Accili, and Alan R. Tall
}

Department of Medicine, Columbia University, New York, New York, USA.

\begin{abstract}
Type 2 diabetes is associated with accelerated atherogenesis, which may result from a combination of factors, including dyslipidemia characterized by increased VLDL secretion, and insulin resistance. To assess the hypothesis that both hepatic and peripheral insulin resistance contribute to atherogenesis, we crossed mice deficient for the LDL receptor ( $\mathrm{Ldlr}^{-/}$mice) with mice that express low levels of IR in the liver and lack IR in peripheral tissues (the $\mathrm{L1}^{\mathrm{B} 6}$ mouse strain). Unexpectedly, compared with $L d l r^{-/-}$controls, $L 1^{B 6} \mathrm{Ldlr}^{-/-}$mice fed a Western diet showed reduced VLDL and LDL levels, reduced atherosclerosis, decreased hepatic AKT signaling, decreased expression of genes associated with lipogenesis, and diminished VLDL apoB and lipid secretion. Adenovirus-mediated hepatic expression of either constitutively active AKT or dominant negative glycogen synthase kinase (GSK) markedly increased VLDL and LDL levels such that they were similar in both $\mathrm{Ldlr}^{-/-}$and $L 1^{B 6} \mathrm{Ldl}^{-/-}$mice. Knocking down expression of hepatic IR by adenovirus-mediated shRNA decreased VLDL triglyceride and apoB secretion in $\mathrm{Ldlr}^{-/-}$mice. Furthermore, knocking down hepatic IR expression in either WT or $o b / o b$ mice reduced VLDL secretion but also resulted in decreased hepatic Ldlr protein. These findings suggest a dual action of hepatic IR on lipoprotein levels, in which the ability to increase VLDL apoB and lipid secretion via AKT/GSK is offset by upregulation of Ldlr.
\end{abstract}

\section{Introduction}

Increased atherosclerosis is a major cause of morbidity and mortality in type 2 diabetes and metabolic syndrome (1), but the underlying mechanisms are poorly understood. Although vascular insulin resistance and hyperglycemia are likely to be involved, a fundamental reason for accelerated atherogenesis in diabetes and metabolic syndrome is dyslipidemia, characterized by increased VLDL and reduced HDL levels (2). Increased hepatic VLDL apoB secretion is a characteristic feature of type 2 diabetes, accounting in part for diabetic dyslipidemia $(3,4)$.

Several mechanisms have been proposed to account for the increased VLDL triglyceride ( $\mathrm{Tg}$ ) and apoB secretion in diabetes and metabolic syndrome. Peripheral insulin resistance gives rise to an increased flux of free fatty acids to the liver, resulting in enhanced $\mathrm{Tg}$ synthesis, decreased apoB degradation, and increased secretion of $\operatorname{VLDL}(5,6)$. In cell-culture models, free fatty acids cause a decrease in ER-associated, ubiquitin-dependent apoB degradation (7), and thus, availability of lipid for VLDL synthesis appears to be a key factor regulating apoB synthesis and secretion (8). In addition, insulin treatment has been reported to suppress apoB secretion in cultured cells (9) and to decrease apoB trans-

Conflict of interest: The authors have declared that no conflict of interest exists. Nonstandard abbreviations used: ACC, acetyl-CoA carboxylase; FAS, fatty acid synthase; FoxO1, forkhead box O1; FoxO1-ADA, adenovirus encoding constitutively nuclear FoxO1; FPLC, fast-performance liquid chromatography; GSK, glycogen synthase kinase; GSK-KM, adenoviral vector expressing dominant negative GSK; GSK-S9A, adenoviral vector expressing constitutively active GSK; Igfbp1, IGF-binding protein 1; IR shRNA, adenovirus encoding shRNA sequences against IR mRNA; $L 1^{B 6}$ mice, transgenically rescued $I R^{-/-}(\mathrm{L} 1)$ mice in C57BL/ 6 background; $\mathrm{LiCl}$, lithium chloride; LIRKO, liver-specific IR KO; mTOR, mammalian target of rapamycin; MTP, microsomal triglyceride transfer protein; myrAKT, adenoviral vector expressing myristoylated Akt; PEPCK, phosphoenolpyruvate; SCD1, stearoyl-CoA desaturase; SREBP, sterol regulatory element-binding protein; Tg, triglyceride; TTR, transthyretin; WTD, Western diet.

Citation for this article: J. Clin. Invest. doi:10.1172/JCI36523. lation (10), and an analysis of insulin signaling in hepatocytes derived from insulin-resistant, fructose-fed hamsters (11) as well as some correlative human data suggest that there are direct effects of insulin in hepatocytes to suppress apoB and VLDL secretion in vivo $(12,13)$. Thus, decreased insulin signaling in the periphery and liver could both act to increase VLDL secretion.

Mice with genetic knockouts of key molecules in the insulin signaling pathway have provided an important tool for elucidating the effects of decreased insulin signaling in peripheral tissues and liver (14-16). $I R^{-/-}$mice die in the neonatal period from diabetic ketoacidosis but can be rescued by low-level expression of the IR in the liver, brain, and pancreas (the L1 mouse strain). Characterization of L1 mice on a mixed genetic background showed marked hyperinsulinemia and moderately increased hepatic gluconeogenesis $(17,18)$. We bred the L1 strain into a uniform C57BL/6 background ( $L 1^{B 6}$ mice) in order to study the effects of decreased insulin signaling in various tissues on atherogenesis.

Transplantation of bone marrow from $L 1^{B 6}$ mice into $L d l^{-/}$recipients, followed by Western diet (WTD) feeding led to a moderately increased atherogenesis, owing primarily to increased macrophage apoptosis (19). In the present study, we assessed atherogenesis in the $L 1^{B 6} \mathrm{Ldlr}^{-1}$ strain that had been used for bone marrow donation in our earlier study. Since these mice had decreased levels of IRs in liver and no IRs in arteries, peripheral tissues, and bone marrow cells, we thought they would have dramatically increased atherosclerosis compared with $\mathrm{Ldll}^{-/}$controls. To our surprise, we found that they were protected from atherosclerosis, reflecting reduced plasma levels of VLDL and LDL and reduced hepatic VLDL secretion. This led to studies showing a direct relationship between hepatic insulin signaling via the IR and regulation of VLDL apoB and lipid secretion. In addition to using the $L 1^{B 6} \mathrm{Ldlr}^{-/}$strain for these studies, we also confirmed key findings by direct knockdown of the hepatic IR using adenovirus carrying shRNA sequences targeting IR mRNA (IR shRNA). 
Table 1

Metabolic characteristics of $L d l r^{-1}$ and $L 1^{B 6} L d l r^{-1}$ mice

\begin{tabular}{|c|c|c|c|}
\hline Genotype & & Chow & WTD \\
\hline \multirow[t]{7}{*}{$L d l r^{-}$} & Body weight (g) & $23.0 \pm 0.3$ & $29.5 \pm 0.7$ \\
\hline & Glucose (mg/dl) & $167.5 \pm 12.2$ & $202.0 \pm 11.4$ \\
\hline & Insulin (pg/ml) & $1016.5 \pm 126$ & $1741.5 \pm 185$ \\
\hline & Cholesterol (mg/dl) & $181.7 \pm 10.1$ & $992.7 \pm 43.9$ \\
\hline & HDL-C (mg/dl) & $79.8 \pm 10.7$ & $87.1 \pm 4.2$ \\
\hline & $\operatorname{Tgs}(\mathrm{mg} / \mathrm{dl})$ & $81.9 \pm 7.1$ & $587 \pm 41.6$ \\
\hline & FFA $(\mathrm{mmol} / \mathrm{l})$ & $1.45 \pm 0.2$ & $1.78 \pm 0.3$ \\
\hline \multirow[t]{7}{*}{$L 1^{B 6} L d l r^{-}-$} & Body weight (g) & $21.1 \pm 0.8$ & $29.5 \pm 0.5$ \\
\hline & Glucose (mg/dl) & $118.4 \pm 12.9^{B}$ & $195.3 \pm 21.5$ \\
\hline & Insulin (pg/ml) & $4901.7 \pm 520^{A}$ & $9233.6 \pm 75.6^{\mathrm{A}}$ \\
\hline & Cholesterol (mg/dl) & $131.1 \pm 9.4^{\mathrm{B}}$ & $664.2 \pm 66.2^{A}$ \\
\hline & HDL-C (mg/dl) & $60.4 \pm 9.1$ & $79 \pm 2.9$ \\
\hline & $\operatorname{Tgs}(\mathrm{mg} / \mathrm{dl})$ & $75.8 \pm 6.1$ & $368.2 \pm 37.5^{A}$ \\
\hline & $\mathrm{FFA}(\mathrm{mmol} / \mathrm{l})$ & $1.15 \pm 0.3$ & $2.07 \pm 0.4$ \\
\hline
\end{tabular}

Metabolic characteristics were measured in plasma from mice fasted for 5 hours. Mice were fed on chow diet ( 8 weeks old) or on WTD for 10 weeks (18 weeks old). Values are expressed as mean \pm SEM. $n=10-15 ;{ }^{A} P<0.001 ;{ }^{B} P<0.01$. HDL-C, HDL cholesterol.

\section{Results}

Metabolic parameters in $L 1^{B 6} \mathrm{Ldlr} /-$ mice. We initially assessed metabolic parameters, atherogenesis, and plasma lipoprotein changes in $\mathrm{L}^{\mathrm{B} 6} \mathrm{Ldll}^{-/}$and $\mathrm{Ldlr^{-/ }}$ control mice in a uniform C57BL/ 6 background (Table 1). Compared with $\mathrm{Ldlr}^{-/}$controls on the chow diet, $L 1^{B 6} \mathrm{Ldlr}^{-/}$mice had about 5-fold higher insulin levels. In contrast with L1 mice in a mixed genetic background (17), the $L 1^{B 6} \mathrm{Ldlr}^{-/}$strain had slightly lower glucose levels (Table 1 ) and showed increased insulin sensitivity during a glucose-tolerance test (Supplemental Figure 1A; supplemental material available online with this article; doi:10.1172/JCI36523DS1). At 8 weeks of age, male mice were switched from chow to WTD and maintained on the diet for 12 weeks before sacrifice for atherosclerosis studies. On the WTD, both groups developed higher insulin and glucose levels, but $L 1^{B 6} \mathrm{Ldlr}^{-/}$mice maintained 5-fold higher insulin levels than controls, while glucose levels and glucose tolerance test responses were similar in the 2 groups (Supplemental Figure 1B). Plasma free fatty acid levels were similar in the 2 groups of mice (Table 1 ), as reported previously in nonobese mice with peripheral insulin resistance $(17,20)$. Plasma adiponectin levels were modestly increased in $L 1^{B 6} \mathrm{Ldlr}^{-/}$mice $(8.6 \pm 0.5 \mu \mathrm{g} / \mathrm{ml}$ vs. $11.1 \pm 0.9 \mu \mathrm{g} / \mathrm{ml}$, $P=0.04)$, similar to those in a previous report (21).

Atherosclerosis studies. After 12 weeks on the WTD, there was an approximately $40 \%$ reduction in proximal aortic atherosclerosis in $L 1^{B 6} \mathrm{Ldlr}^{-/}$mice compared with $L d l r^{-/}$controls (Figure $1 \mathrm{~A}$ ), as measured by lesion area. Western blot analysis showed absence of IR protein expression in the aortae of $L 1^{B 6} \mathrm{Ldlr}^{-1}$ mice (Figure 1B). Evaluation of lesion morphology indicated that lesions were smaller but had relative necrotic core areas similar to those in the former group $(12.3 \% \pm 2.8 \%$ vs. $15.4 \% \pm 1.7 \%, P=0.3)$. Quantification of total hepatic IR protein using an antibody that recognizes both endogenous and transgenic IR showed more than $90 \%$ reduction in levels in $L 1^{B 6} \mathrm{Ldlr}^{-1}$ mice. We have previously reported that IR transgene expression was approximately $50 \%$ of normal in mice of mixed genetic background $(17,18,21)$. Thus, backcross-

\section{Figure 1}

Quantification of proximal aortic lesions, aortic insulin receptor protein levels, and correlation between hepatic insulin receptor protein levels and atherosclerotic lesion area. (A) Dot plot showing the aortic root lesion area of individual $\mathrm{LdIr}^{-1-}(n=15)$, heterozygote

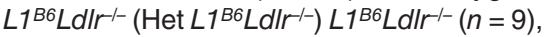
and $L 1^{B 6} L d l r^{--}(n=14)$ male mice after 12 weeks on the WTD. Horizontal bars represent the mean in each group. (B) Western blot analysis of IR protein levels in proximal aortae from 10 -week-old $L d / r^{-1}$ and $L 1^{B 6} \mathrm{Ldll}^{-1}$ male mice. (C) Linear correlation plot showing mean atherosclerotic lesion areas from $\mathbf{A}$ and mean of hepatic insulin receptor protein from 3 mice of indicated genotypes. Order of the indicated genotypes from left to right are $L 1^{B 6} \mathrm{Ldll}^{-1}$ - plus TTR-IR (+TTR) (open circle), heterozygote $L 1^{B 6} \mathrm{LdIr}^{-1}$ minus TTR-IR (-TTR) (triangle), heterozygote $L 1^{B 6} L d l r^{-1}$-plus TTR-IR (diamond), Ldlr-l-plus TTR-IR (square), and $\mathrm{LdII}^{-1-}$ minus TTR-IR (filled circle). Data represent mean $\pm \mathrm{SEM} . R^{2}$, correlation coeffcient.
A

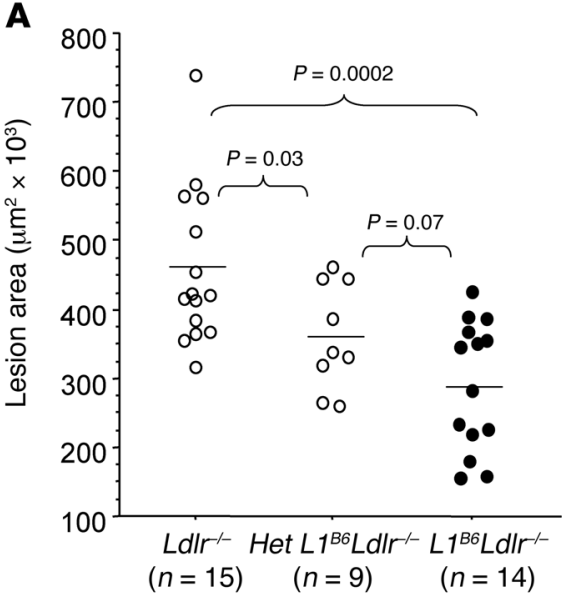

C

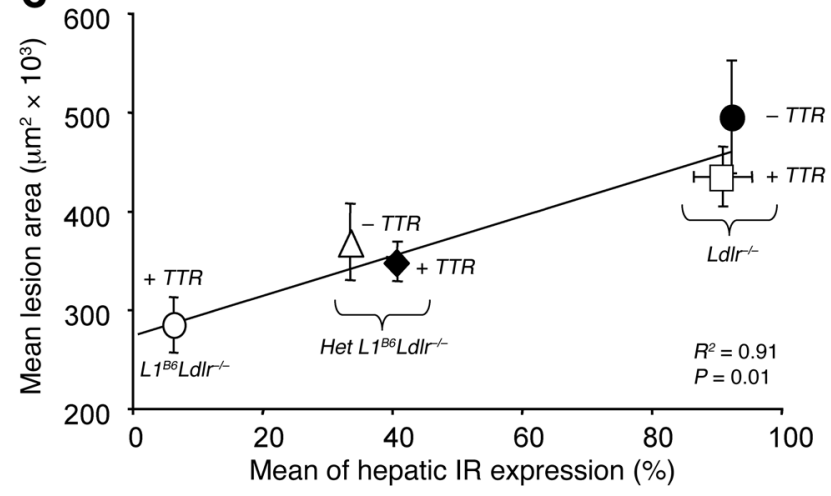


A
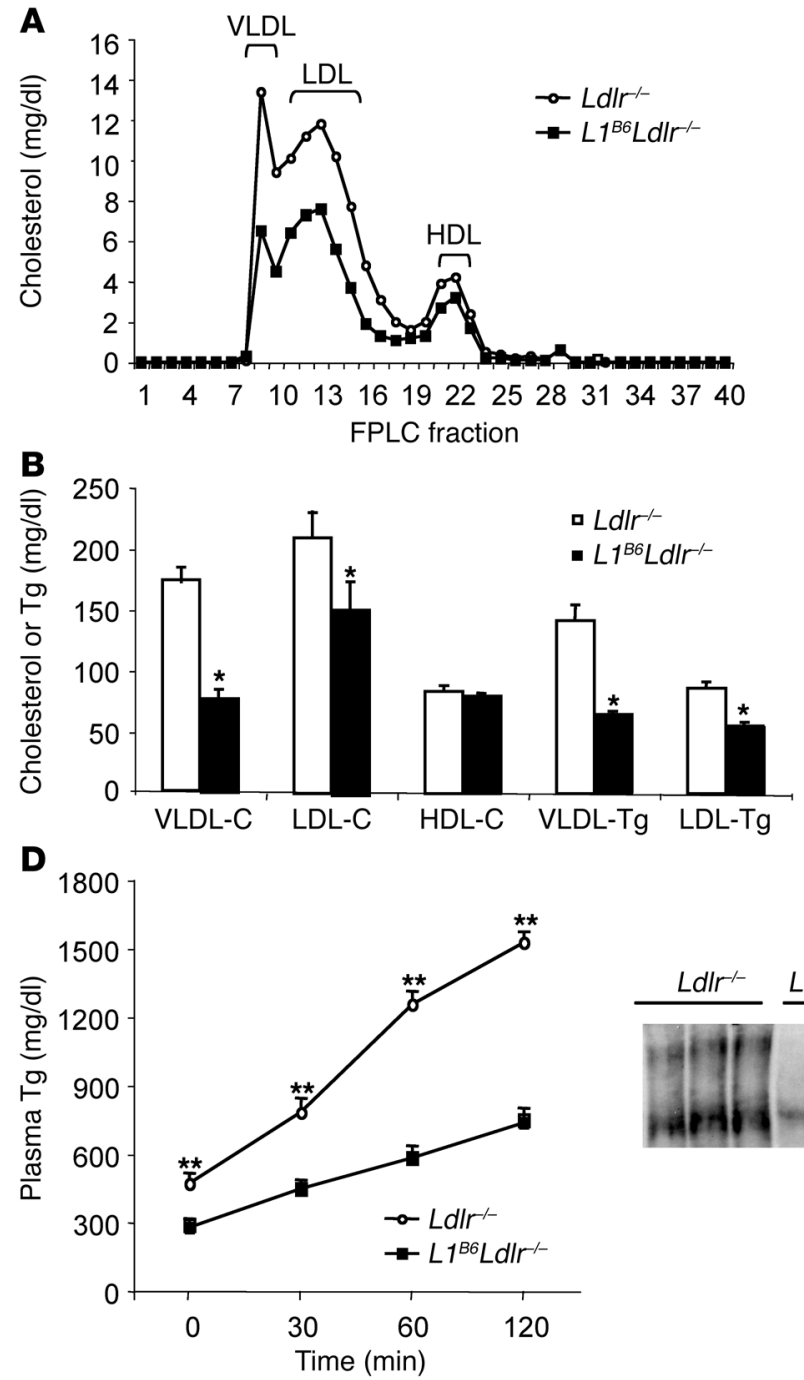

C
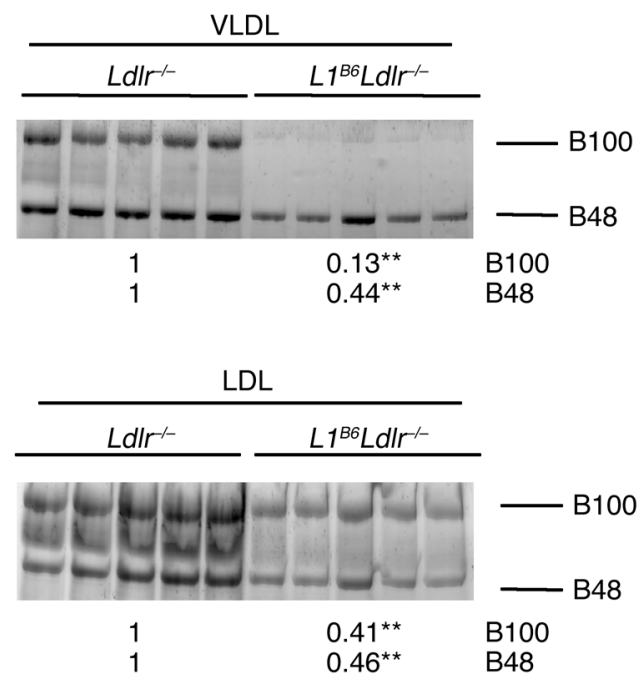

Figure 2

Plasma lipoprotein profiles, plasma lipoprotein cholesterol, Tg and apoB levels, and $\mathrm{Tg}$ and apoB production of $L d l r^{-1}$ and $L 1^{B 6} L d l r^{1-}$ mice. (A) Lipoprotein separation by FPLC. Pooled plasma from $10 \mathrm{Ldll}^{-1-}$ (circles) and $10 \mathrm{~L}^{\mathrm{B}} \mathrm{Ldll}^{-1}$ (squares) mice described in Figure $1 \mathrm{~A}$ were used for FPLC analysis. Plasma was collected after 5 hours of fasting. Fractions were used to quantify plasma lipoprotein cholesterol concentration. (B) Quantification of plasma VLDL cholesterol (VLDL-C), LDL cholesterol (LDL-C), HDL cholesterol (HDL-C), VLDL-Tg, and LDL-Tg from $L d l r^{-1}$ (white bars) and $L 1^{B 6} L d l r^{-1-}$ (black bars) mice. Plasma was collected from mice fed WTD for 2 weeks, after a 5-hour fast $(n=13-14$; $\left.{ }^{\star} P<0.001\right)$. (C) Representative sample of apoB (apoB100 [B100] and apoB48 [B48]) levels in Ldlr $r^{-1-}$ and $L 1{ }^{B 6} L d l r^{-1-}$ mice shown in B. apoBcontaining lipoproteins (VLDL and LDL) isolated by ultracentrifugation were resolved by SDS-PAGE and visualized by Coomassie blue staining. Each lane represents an individual mouse. apoB was normalized to the amount in the $L d l r^{-1}$ controls $\left(n=5\right.$; $\left.{ }^{\star *} P<0.01\right)$. (D) Tg and apoB production in $\mathrm{Ldll}^{-1}$ and $L 1^{B 6} \mathrm{Ldll} \mathrm{r}^{-1}$ mice. Tg production was determined by measuring plasma $\mathrm{Tg}$ concentrations at indicated times after Triton WR-1339 injection $(n=4-5)$. Equal amounts of plasma from each mouse 2 hours after injection with ${ }^{35}$ S-methionine was resolved by SDS-PAGE to visualize ${ }^{35} \mathrm{~S}$-methionine-labeled apoB proteins. Similar results were obtained from 2 independent experiments.

ing onto the BL6 background resulted in lower transgene expression levels. This is likely an effect of transgene methylation (22) or of modifier genes (23). There was also a significant decrease in atherosclerosis in haploinsufficient (i.e., $I R^{+-}$) $L 1^{B 6} L d l r^{--}$mice with about $40 \%$ IR protein levels in liver (15) compared with that in $L d l r^{-/}$mice (Figure 1A). Regression analysis of hepatic IR levels versus atherosclerotic lesion area in these different groups of mice revealed a strong direct relationship between IR levels and atherosclerotic lesion areas (Figure $1 C ; R^{2}=0.91$, where $R^{2}$ is the correlation coefficient; $P=0.01)$. This analysis also included atherosclerotic lesion areas in $\mathrm{Ldlr}^{--}$and heterozygote $L 1^{B 6} \mathrm{Ldlr^{-/ }}$ mice with and without the transthyretin-IR (TTR-IR) transgene, showing that atherosclerotic lesion area was unaffected by the presence of the IR transgene (Figure 1C).

Plasma lipoprotein analysis. An explanation for these findings emerged from the analysis of plasma lipoprotein profiles. Fastperformance liquid chromatography (FPLC) separation showed a substantial reduction of VLDL and LDL cholesterol levels in WTDfed $L 1^{B 6} L d l r^{-/}$mice compared with those in $L d l r^{-1}$ controls, with no difference in HDL levels (Figure 2A). There were no differences in hepatic SR-B1 expression, lecithin-cholesterol acyltransferase (LCAT) activity, and lipoprotein lipase (LPL) activity between these 
A
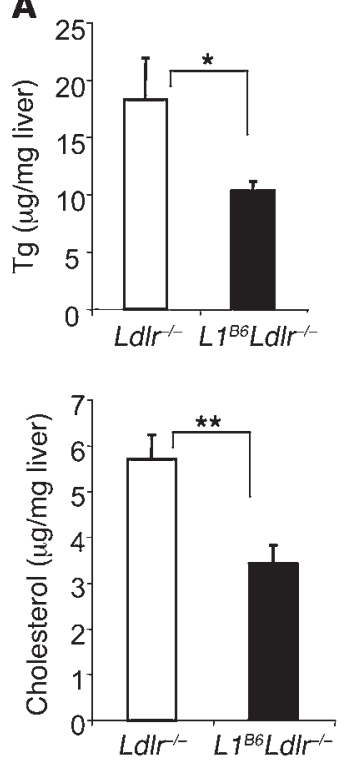

B

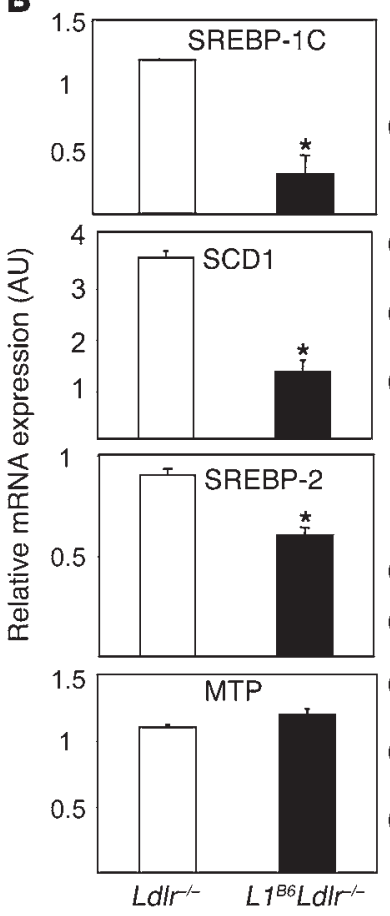

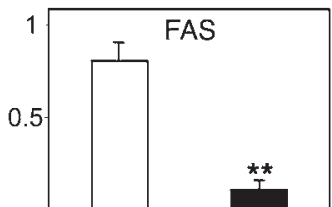
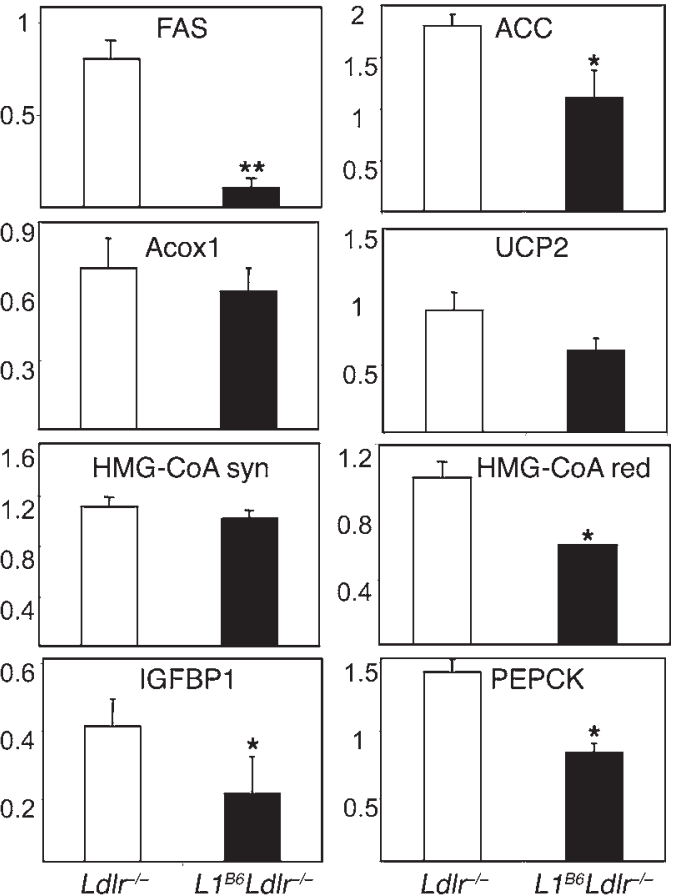

Figure 3

Hepatic lipid content and mRNA expression of genes involved in lipid metabolism. (A) Hepatic Tg and cholesterol (Chol) levels in the livers of $\mathrm{LdIr}^{--}$and $L 1^{B 6} \mathrm{LdIr^{- } -}$ mice $(n=4-5)$. Livers were collected from mice fed WTD for 2 weeks, after a 5-hour fast. (B) Analysis of hepatic gene expression by real-time quantitative PCR in $L d l r^{-1}$ (white bars) and $L 1^{B 6} L d l r^{-1}$ (black bars) mice $(n=3)$. Livers were collected from mice fed WTD for 2 weeks. Acox1, acyl-CoA oxidase 1; UCP2, uncoupling protein 2; syn, synthase; red, reductase. ${ }^{*} P<0.05 ;{ }^{* *} P<0.01$.

2 groups (Supplemental Figure 1, C, E, and F). Analysis of plasma lipoprotein changes revealed similar findings in $\mathrm{L}^{B 6} \mathrm{Ldlr}^{-/}$mice that were heterozygous for the IR null allele (i.e., these mice had IR levels that were intermediate between those of $\mathrm{Ldlr}^{/-}$and $\mathrm{L1}^{B 6} \mathrm{Ldlr^{-/ }}$ mice), with reduced VLDL and LDL cholesterol levels compared with those in $\mathrm{Ldlr}^{/-}$mice (not shown). These data establish a gene-dosage correlation between IR levels and VLDL and LDL cholesterol levels. These findings were confirmed by ultracentrifugal isolation of lipoprotein fractions, revealing approximate $55 \%$ and $28 \%$ decreases in VLDL and LDL cholesterol levels as well as significant reductions in the apoB content of VLDL and LDL, respectively (Figure 2, B and C). To determine whether these changes resulted from altered hepatic lipoprotein secretion, we carried out a Triton WR-1339 injection study. Triton WR-1339 blocks the removal of lipoproteins from the circulation, and thus the rate of accumulation of lipoprotein components in plasma reflects secretion rate (24). These studies revealed a marked reduction in the secretion of Tgs and VLDL cholesterol as well as apoB100 and apoB48 in the $\mathrm{L1}^{\mathrm{B} 6} \mathrm{Ldlr^{-/ }}$ mice (Figure 2D and Supplemental Figure 1D). There were also reduced levels of Tgs and cholesterol in the livers of these mice (Figure 3A).

$L 1^{B 6} \mathrm{Ldlr}^{-/}$mice show decreased lipogenic gene expression. The decrease of hepatic $\mathrm{Tg}$ and cholesterol content and the reduced VLDL secretion in $L 1^{B 6} L d l r^{-1}$ mice could potentially arise from decreased expression of lipogenic genes in the liver. We found a profound decrease in the expression of sterol regulatory element-binding protein isoform-1c (SREBP-1c) mRNA in fed liver samples from $L 1^{B 6} \mathrm{Ldlr}^{--}$mice compared with $\mathrm{Ldlr}^{-/}$controls, accompanied by reduced expression of lipogenic genes such as fatty acid synthase
(FAS), acetyl-CoA carboxylase (ACC), and stearoyl-CoA desaturase $(S C D)$, and decreased hepatic Tg and cholesterol content (Figure 3, $A$ and B). HMG-CoA reductase showed modestly decreased expression, while expression of HMG-CoA synthase was unchanged. The expression of genes mediating fatty acid oxidation (Figure $3 \mathrm{~B}$ ) was unchanged in $\mathrm{L1}^{B 6} \mathrm{Ldlr}^{-/}$compared with $\mathrm{Ldlr^{-/ }}$ mice. L-pyruvate kinase, a key target gene of ChREBP - another transcriptional regulator of lipogenic genes - did not show altered expression (not shown). Other potential candidate molecules involved in the regulation of VLDL levels, including apoB, microsomal Tg transfer protein (MTP), and LPL, were unchanged (Figure 3B and data not shown). Consistently, $L 1^{B 6} \mathrm{Ldl}{ }^{-/-}$mice showed decreased FAS and SCD1 protein expression compared with control $\mathrm{Ldlr}^{-/}$mice, as expected, while there was no difference in MTP protein levels between groups (data not shown and Supplemental Figure 1C). Consistent with previous findings (18), the expression of gluconeogenetic gene G6Pase was unaltered while the expression of IGFbinding protein 1 (Igfbp1) and phosphoenolpyruvate carboxykinase (PEPCK) was significantly reduced in $L 1^{B 6} \mathrm{Ldlr}^{-/-}$(data not shown and Figure 3B). Together, these observations suggest a picture of reduced insulin stimulation of lipogenic gene expression without major alteration in gluconeogenetic gene expression.

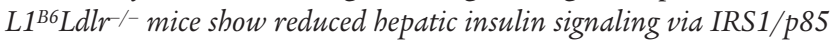
and $A K T$. In order to define the mechanisms of this mixed picture of insulin resistance and sensitivity, we monitored steady state levels of hepatic insulin signaling in the 2 groups of mice. Hepatic IR protein levels were markedly reduced $(\geq 90 \%)$ in $L 1^{B 6} \mathrm{Ldlr} /-$ mice (Figure 4A). While IRS1 protein was increased, the association 
A

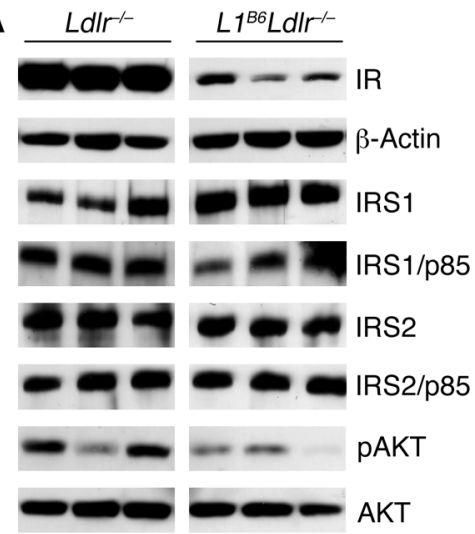

\begin{tabular}{l} 
Fold change \\
\hline$\times 0.1^{* *}$ \\
$\times 1.1$ \\
$\times 1.6^{\star}$ \\
$\times 0.5^{*}$ \\
$\times 1.0$ \\
$\times 0.9$ \\
$\times 0.3^{*}$ \\
$\times 0.9$
\end{tabular}

B

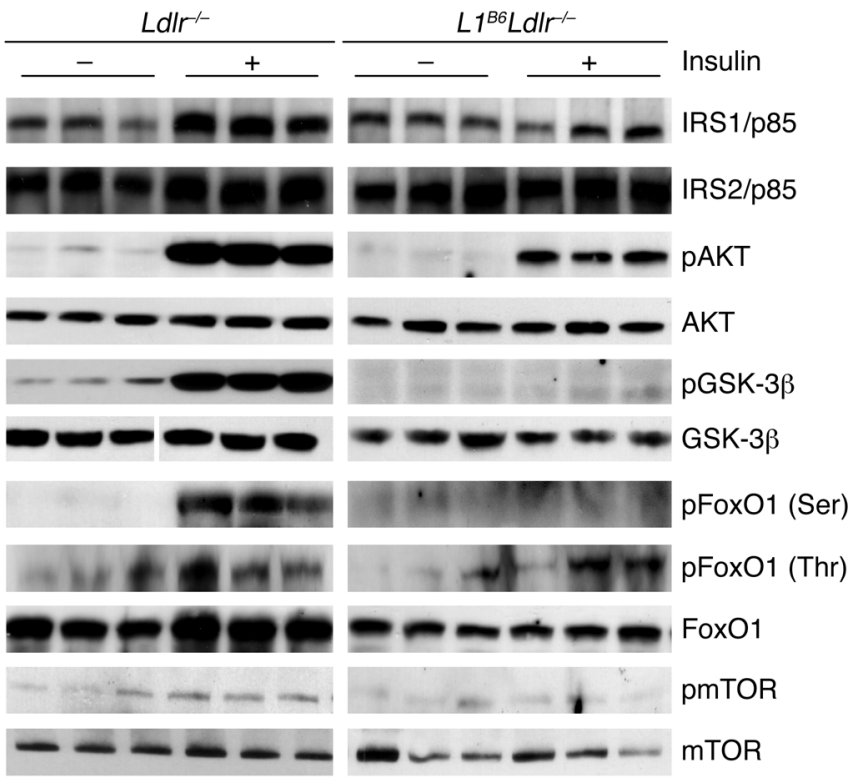

Figure 4

Hepatic expression of insulin signaling molecules in $L d l r^{-1-}$ and $L 1^{B 6} L d l r^{-1-}$ mice. (A) Western blot analysis of IR ( $\beta$-subunit), $\beta$-actin, IRS1, IRS1-associated p85 (regulatory subunit of PI3K), IRS2, IRS2-associated p85, pAKT, and AKT from $L d l r^{-1-}$ and $L 1^{186} L d l r^{-1-}$ mouse livers of male mice after 2 weeks on WTD. Mice were fasted for 5 hours. Fold change shows the expression ratio of $L 1^{B 6} L d l r^{-1-}$ over $L d l r^{-1}$. $n=3$; ${ }^{*} P<0.05$; ${ }^{* *} P<0.01$. (B) Western blot analysis of hepatic expression of indicated signaling proteins following acute i.p. injection of an insulin bolus (+) or saline (-) into $\mathrm{Ldll}^{--}$and $\mathrm{L}^{\mathrm{B}}{ }^{\mathrm{L}} \mathrm{Ldl} \mathrm{r}^{-/}$mice fasted for 16 hours. Lanes were run on the same gel but were noncontiguous.

of the p85 regulatory subunit of PI3 kinase with IRS1 and AKT phosphorylation was reduced in $L 1^{B 6} \mathrm{Ldlr}^{-/}$mice compared with $\mathrm{Ldlr}^{-/-}$mice. The levels of IRS2 protein and its association with the regulatory $\mathrm{p} 85$ subunit of $\mathrm{PI} 3 \mathrm{~K}$ were comparable in the 2 groups of mice. Phospho-AKT (Ser 473) was reduced by 3 -fold $(P<0.05)$ in $L 1^{B 6} \mathrm{Ldlr}^{-/}$mice compared with that in $\mathrm{Ldlr}{ }^{-/}$controls.

To further dissect the effects of IR deficiency on the insulin signaling pathway, fasted mice were injected with a bolus of insulin (Figure 4B). This resulted in an increased association of the regulatory p85 subunit of PI3 kinase with IRS1 and IRS2 in Ldlr-/mice and an increase in AKT phosphorylation. These responses were markedly blunted in $\mathrm{L1}^{B 6} \mathrm{Ldlr^{-/ }}$ mice. Furthermore, the insulin-induced phosphorylation of AKT substrates, notably glycogen synthase kinase 3 (GSK3), forkhead box O1 (FoxO1), and mammalian target of rapamycin (mTOR), was markedly attenuated in $L 1^{B 6} \mathrm{Ldlr}^{-/-}$mice compared with $\mathrm{Ldlr^{-/ }}$ controls (Figure 4B). Phosphorylation of FoxO1 on Ser253, the canonical AKT site (25), was impaired, while phosphorylation at Thr24, which is AKT-independent $(26,27)$, was maintained in $L 1^{B 6} \mathrm{Ldll}^{-/-}$mice. Together, these findings suggest that reduced levels of hepatic IR in $L 1^{B 6} \mathrm{Ldlr}{ }^{-/}$mice lead to reduced insulin signaling via IRS1/PI3K and reduced levels of AKT phosphorylation and its downstream targets, leading to reduced expression of lipogenic genes and decreased VLDL secretion.

Knockdown of hepatic IRs by shRNA impairs VLDL secretion. In order to further evaluate the direct effects of hepatic insulin signaling on VLDL apoB and lipid secretion, we used adenovirus-mediated IR shRNA (IR shRNA) to knock down expression of the IR in WTD-fed $\mathrm{Ldlr}^{-1-}$ mice. Ten days after injection of IR shRNA, there was significantly reduced $(\sim 80 \%)$ hepatic IR protein expression in $\mathrm{Ldll}^{-/}$- mice compared with mice injected with a control scrambled shRNA adenovirus (Figure 5A). Similarly, AKT phosphorylation was reduced in IR shRNA-injected mice (Figure 5A), as were plasma and hepatic Tg levels (Supplemental Table 1). There were no differences in body weight, plasma glucose, total or HDL cholesterol levels, or hepatic MTP or apoB mRNA levels between the 2 treatment groups. There was significantly decreased VLDL Tg, cholesterol, and apoB secretion in IR shRNA-treated mice compared with controls (Figure $5, \mathrm{C}-\mathrm{F} ; n=3$ or 4 ). Thus, we confirmed that reduced hepatic insulin signaling leads to decreased VLDL lipid and apoB production.

$A K T$ overexpression increases hepatic lipogenesis and increases VLDL and LDL cholesterol and apoB levels. To further evaluate the role of the insulin signaling pathway in regulation of lipoprotein levels, constitutively active adenovirus expressing myristoylated Akt (myrAKT) or control empty adenoviruses were injected via tail vein into WTD-fed $L 1^{B 6} \mathrm{Ldlr}^{-/-}$and $\mathrm{Ldlr^{-/ }}$ mice (Figure 6). This resulted in increased levels of VLDL and LDL cholesterol and Tgs as well as apoB100 and apoB48 in both groups (Figure 6, A and $\mathrm{B})$. These experiments were also performed in chow-fed mice with similar results (Supplemental Figure 2). We also carried out a dose-response experiment. This showed a clear dose relationship between myrAKT and apoB levels, with increases in apoB seen even at the lowest doses of myrAKT (Figure 6C and Supplemental Figure 2C). Notably, a moderate 3-fold increase in AKT phosphorylation comparable to the levels seen in fasting $o b / o b$ mice (see below) resulted in increased apoB levels (Figure 6C and Supplemental Figure 2C). There was also a marked increase in VLDL lipid and apoB secretion following myrAKT injection in

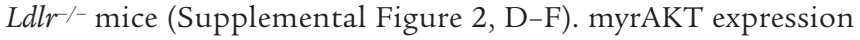
largely eliminated the differences in lipogenic gene expression and lipoprotein levels between the 2 groups, consistent with the hypothesis that reduced insulin signaling via AKT was primarily responsible for altered lipogenic gene expression and reduced VLDL and LDL levels in $\mathrm{L}^{B 6} \mathrm{Ldlr}^{-/-}$mice. 
A
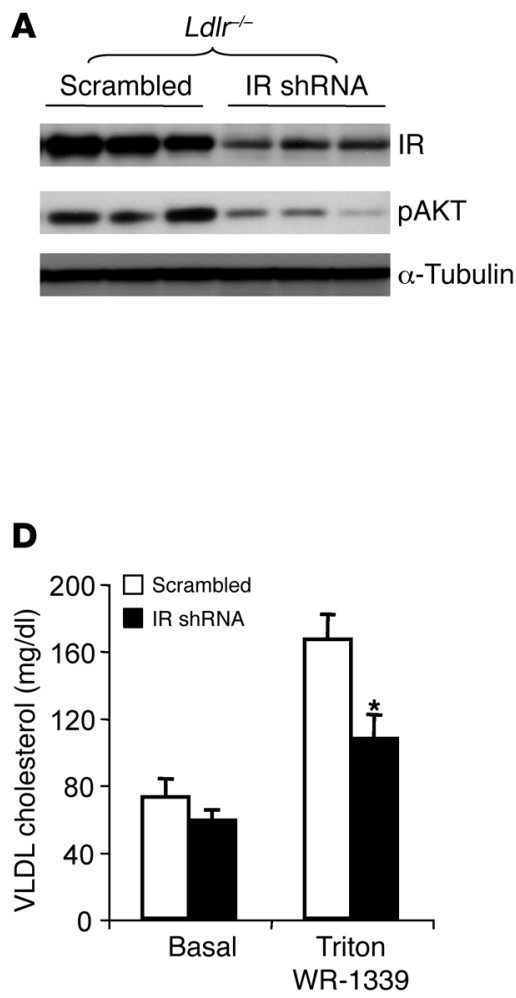

B

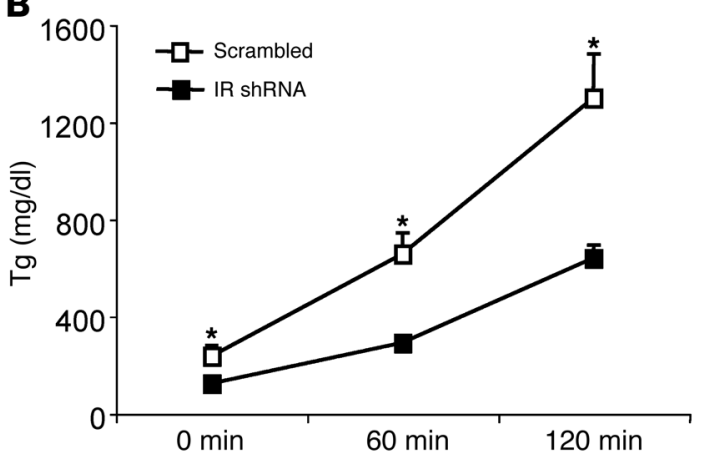

E

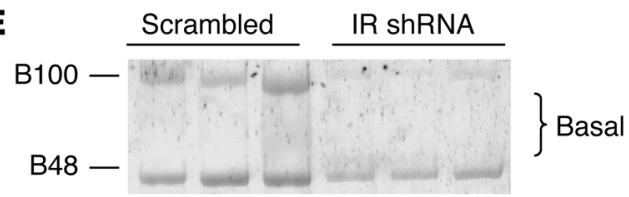

B100

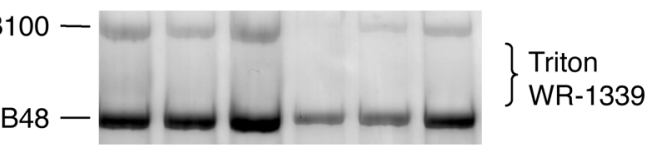

C
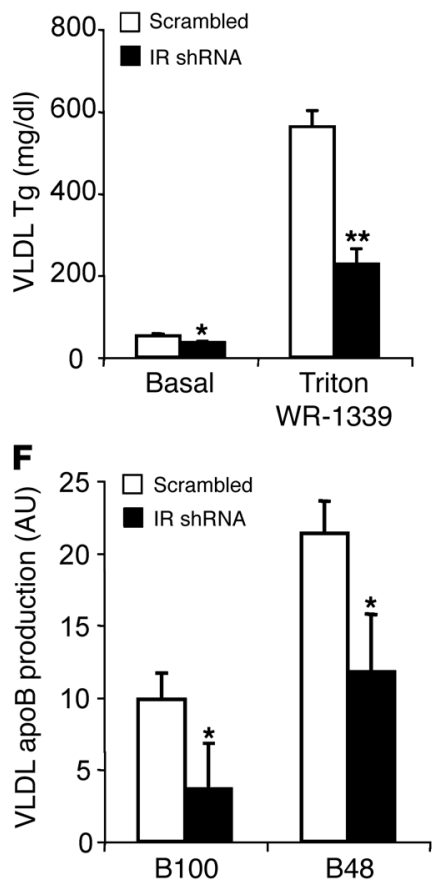

Figure 5

Effect of hepatic IR knockdown on VLDL and apoB production in LdIr-- mice. All mice were 10 to 11 weeks old and had been fed WTD for 2 weeks when virus was injected. (A) Western blot analysis of IR, pAKT, and $\alpha$-tubulin in liver lysates from Ldlr ${ }^{-1}$ mice after infection with scrambled control adenovirus or IR shRNA. Livers were collected after a 5-hour fast. Each lane shows the liver lysate of a different mouse. (B) Tg production in WTD fed Ldlr/- mice treated with control adenovirus (open squares) or IR shRNA (filled squares). Tg production was determined at indicated times after Triton WR-1339 injection. Plasma from each mouse at 0- and 120-minute time points was used for ultracentrifugation to obtain the VLDL fraction. VLDL Tg (C) and VLDL cholesterol (D) secretion were measured. VLDL apoB (apoB100 and apoB48) levels (E) and secretion $(\mathbf{F})$ were examined in control adenovirus- and IR shRNA-treated mice from experiment shown in $\mathbf{B} . n=3-4 ;{ }^{*} P<0.05 ;{ }^{*} P<0.01$.

GSK and regulation of VLDL apoB secretion. We next conducted an analysis of the role of the insulin signaling pathway in the regulation of apoB lipoprotein levels and secretion. We considered the potential involvement of the 3 major pathways acting downstream of AKT, i.e., mTOR, FoxO1, and GSK3 (28), which showed diminished activity in the $\mathrm{L} 1^{B 6} \mathrm{Ldlr^{-1 }}$ mice (Figure 4B). AKT phosphorylates and leads to nuclear exclusion of FoxO1; thus, if AKT signaling and inactivation of FoxO1 was responsible for increased apoB secretion, hepatic overexpression of constitutively active FoxO1 should lead to a reduction in apoB levels. However, when administered to $\mathrm{Ldlr}^{-/-}$mice, increasing amounts of adenovirus encoding constitutively nuclear FoxO1 (FoxO1ADA) did not change plasma apoB levels (Figure 7A) and Tg production (Figure 7B). We then determined whether direct activation of mTOR by AKT could lead to increased apoB secretion. To this end, we administered the mTOR inhibitor rapamycin in $\mathrm{Ldlr}^{-/}$mice. Contrary to expectations, at the higher doses, the mTOR inhibitor caused an increase in apoB levels and cholesterol (Figure 7, C and D), indicating that signaling via mTOR-1 is unlikely to mediate the effects of AKT on apoB levels. Inhibition of signaling via mTOR-1 is known to activate a feedback loop resulting in increased levels of IRS1 (as in Figure 4A) and increased AKT signaling (29), suggesting that increases in VLDL secretion secondary to mTOR inhibition may have been second- ary to increased AKT signaling via other pathways. AKT signaling also leads to phosphorylation and inactivation of GSK3 (28). Thus, if this pathway were involved, a dominant negative form of GSK3 should mimic the effect of AKT and lead to increased secretion of apoB. Indeed, adenoviral vector expressing a dominant negative form of GSK3 (GSK-KM) caused a marked increase in VLDL and LDL apoB100 and apoB48 and cholesterol and Tg levels in both $L d l r^{-/}$and $L 1^{B 6} \mathrm{Ldlr}^{-/-}$mice (Figure 8). Since these experiments relied on overexpression, we also wished to inhibit endogenous GSK3. Lithium chloride ( $\mathrm{LiCl}$ ) has been shown to inhibit GSK3 by an interaction with endogenous AKT that leads to increased GSK3 phosphorylation (30). Thus, we treated $\mathrm{Ldlr}^{-/}$ mice with LiCl. This led to a significant increase in apoB levels in VLDL $(\times 1.5 ; P=0.0005, n=4)$ and $\operatorname{LDL}(\times 1.3 ; P=0.03, n=4)$. Similar results were obtained with $L 1^{B 6} L d l r^{-/}$mice (not shown). These changes were not as dramatic as those observed with the dominant negative GSK3, probably because $\mathrm{LiCl}$ is a relatively ineffective inhibitor of hepatic GSK3 $(31,32)$. Together, these findings suggest that AKT signaling leads to inactivation of GSK and thus increases VLDL and LDL apoB secretion.

Increased AKT activity and VLDL secretion in ob/ob mice. Obese $o b / o b$ mice are commonly employed as a model of insulin resistance: they have markedly increased insulin levels, lipogenesis, and VLDL secretion and develop increased VLDL and LDL levels and acceler- 

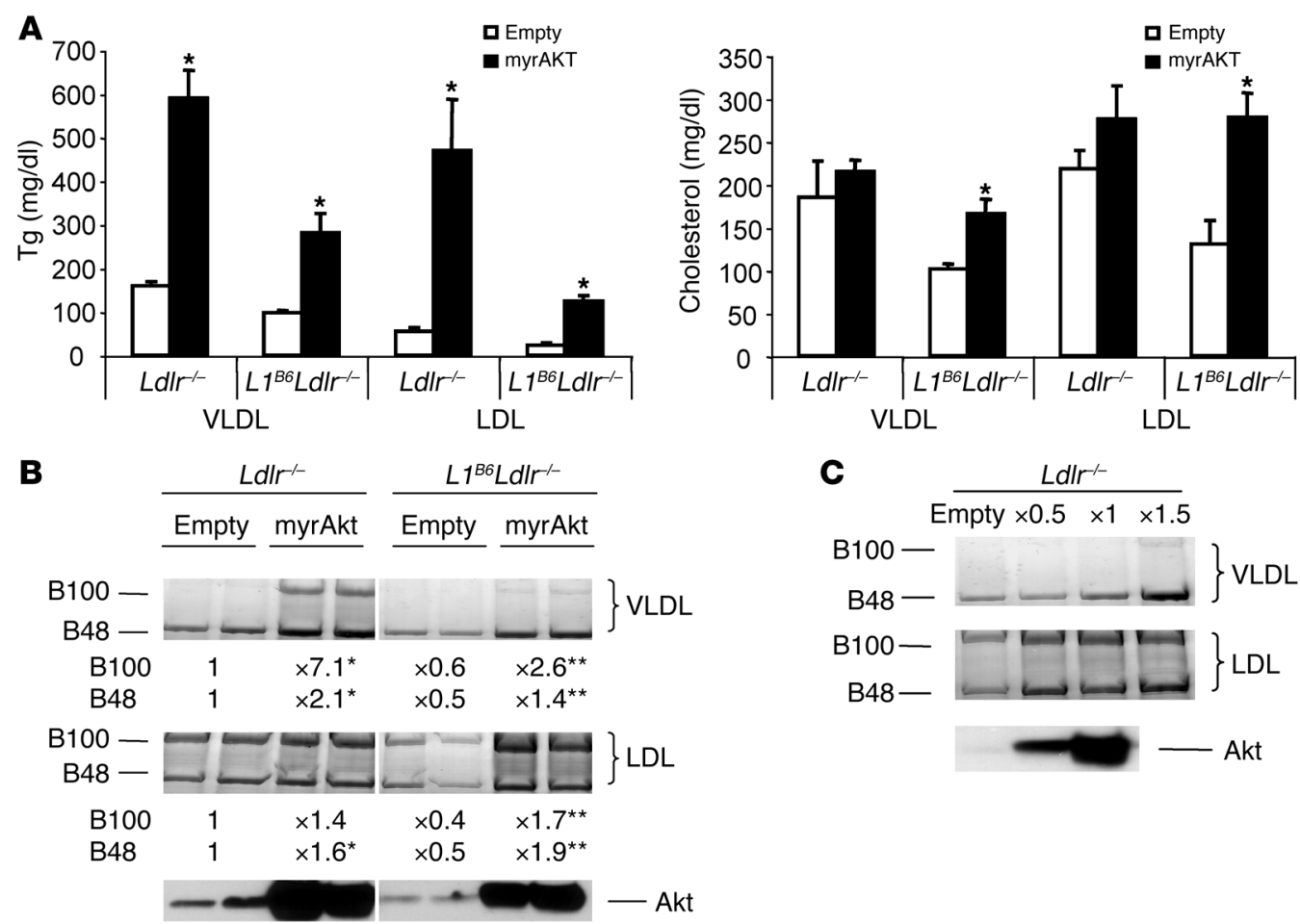

\section{Figure 6}

Plasma lipoprotein lipid and apoB levels in WTD-fed mice injected with myrAKT or control adenovirus. All mice were 10-11 weeks old and fed WTD for 2 weeks before viral injection. (A) Plasma lipoprotein Tg and cholesterol levels in $L d l r^{-1}$ and $L 1^{B 6} L d l r^{-1-}$ mice injected with either control empty adenovirus (white bars) or myrAKT (black bars). Plasma was collected after 5 hours fasting, and VLDL and LDL were separated by ultracentrifugation. (B) apoB contents in VLDL and LDL in mice injected with either control empty adenovirus or myrAKT. apoB-containing lipoproteins were resolved by SDS-PAGE and stained with Coomassie blue. apoB was quantified and normalized to the amount present in the mice carrying control adenovirus. Lowest panel shows Western blot analysis for AKT expression. Representative results are shown. Statistical significance was determined upon comparison of results with those obtained following treatment with the control adenovirus. $n=3$; ${ }^{*} P<0.05$; ${ }^{* *} P<0.01$. All data are representative of at least 2 independent experiments. Lanes were run on the same gel but were noncontiguous. (C) apoB amount in VLDL and LDL in Ldlr ${ }^{-1-}$ mice injected with empty adenovirus or different dosages ( $\times 10^{7} \mathrm{PFU} / \mathrm{g}$ body weight) of myrAKT. Lowest panel shows Western blot analysis of hepatic AKT expression in adenovirus-injected mice.

ated atherogenesis when crossed onto the $L d l r^{/-}$background (33). We found that basal state AKT and GSK3 phosphorylation levels were markedly increased in fasting ob/ob mice (Figure 9A) and were associated with increased VLDL secretion (Figure 9B).

Knockdown of the hepatic IR by shRNA in $o b / o b$ mice resulted in an approximately $90 \%$ reduction in IR protein levels compared with controls (Figure 10A). While there were no differences in body weight, plasma glucose, HDL cholesterol or Tg levels, or hepatic MTP or apoB mRNA levels between mice treated with IR shRNA and scrambled controls, the former mice had higher total plasma cholesterol, increased non-HDL cholesterol (i.e., VLDL+LDL cholesterol), and lower hepatic Tg levels (Supplemental Table 2). Decreased hepatic IR levels resulted in decreased secretion of VLDL Tg and apoB (Figure 10, B-D). However, plasma VLDL and LDL apoB levels were not decreased (Supplemental Figure 4), and as noted, VLDL+LDL cholesterol was increased in $o b / o b$ mice injected with IR shRNA (Supplemental Table 2). This finding was explained by the observation that $o b / o b$ mice had high basal levels of Ldlr protein $(\times 3.2, P=0.0003)$, which were markedly decreased by IR shRNA (Figure 10A). Thus, the decrease in VLDL secretion was offset by a decrease in hepatic Ldlr protein levels, resulting in no change in plasma VLDL and LDL apoB levels and a moderate increase in VLDL+LDL choles- terol levels. Notably, knockdown of IR by shRNA also led to a substantial reduction $(\times 0.4 ; P=0.0005)$ in hepatic Ldlr protein levels in WTD-fed WT mice (Figure 10A).

In order to test the hypothesis that increased AKT signaling led to GSK3 inactivation and thus increased VLDL secretion in $o b / o b$ mice, we administered a low dose of constitutively active GSK3 adenovirus to $o b / o b$ mice. This resulted in decreased secretion of Tg and VLDL apoB following Triton WR-1339 administration in $o b / o b$ mice (Figure 10E). Nuclear SREBP-1c protein was not changed following injection of myrAKT or dominant negative GSK3 viruses (Supplemental Figure 3, A and B). However, there was a modest but significant decrease in nuclear SREBP-1c following injection of constitutively active GSK3 (Supplemental Figure 3C).

\section{Discussion}

We found that $\mathrm{Ldlr}^{-/}$mice with impaired IR expression in liver and lacking IRs in peripheral tissues and aortae had substantially reduced aortic atherosclerosis. Reduced levels of VLDL and LDL in $L 1^{B 6} \mathrm{Ldl}^{-1-}$ mice are very likely responsible for this result. Despite hyperinsulinemia, reduced levels of hepatic IRs led to diminished AKT and GSK3 phosphorylation in $L 1^{B 6} \mathrm{Ldlr^{-1 }}$ mice. Using IR shRNA to knock down the IR, we confirmed that decreased VLDL apoB and lipid secretion were the direct result of reduced 
A

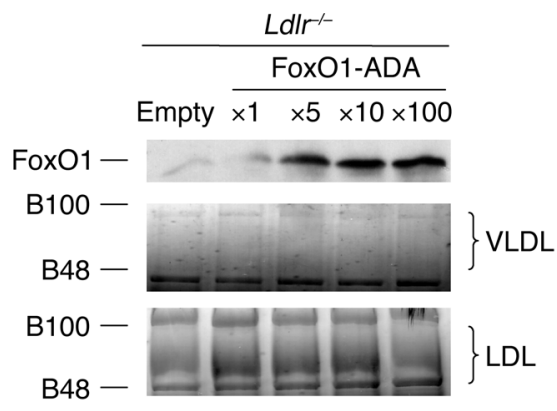

C

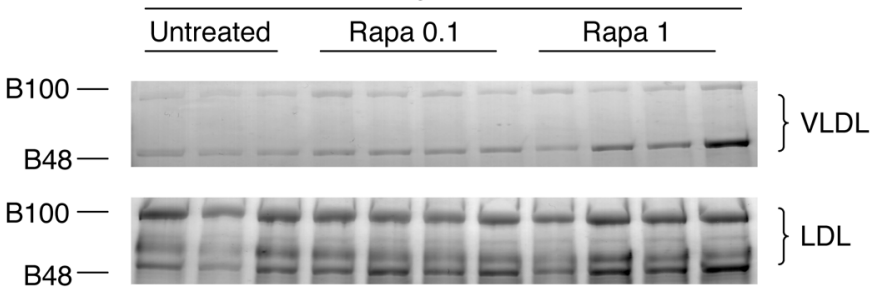

B

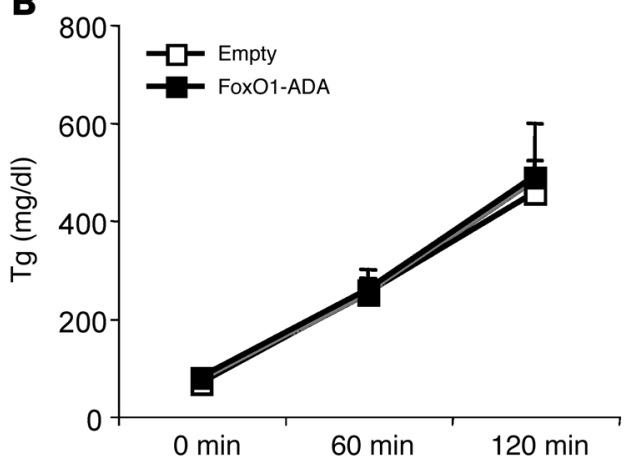

D

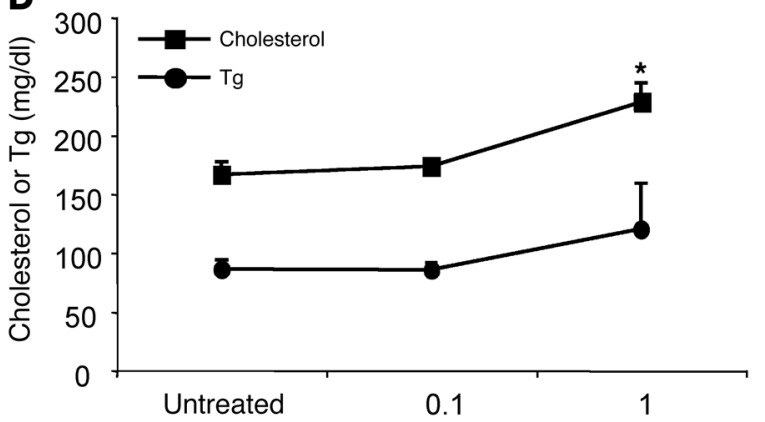

Figure 7

The effect of AKT downstream effectors FoxO1 and mTOR on plasma apoB levels. (A) Plasma VLDL and LDL apoB levels in FoxO1-overexpressing mice. Ten-week-old $L d / r^{-1-}$ mice were injected with empty adenovirus or different dosages ( $\times 10^{7}$ PFU/g body weight) of FoxO1-ADA. Four days after adenovirus injection, livers and plasma samples were collected after a 5-hour fast. Upper panel is representative of Western blot analysis of plasma lipoprotein fractions after hepatic FoxO1 expression. Similar results were obtained in 2 other experiments. (B) Tg production in WTD-fed $\mathrm{Ldll}^{-1-}$ mice treated with control empty adenovirus (open squares) or FoxO1-ADA (filled squares). Tg production was determined at indicated times after Triton WR-1339 injection $(n=4)$. (C) apoB levels in mice treated with mTOR inhibitor (rapamycin [Rapa]). Ldl $r^{-/}$mice fed chow received saline/DMSO with or without rapamycin $(0.1 \mu \mathrm{g} / \mathrm{g}$ or $1 \mu \mathrm{g} / \mathrm{g}$ body weight). (D) Plasma cholesterol (filled squares) and Tg levels (filled circles) from data shown in C. $n=3-4 ;{ }^{*} P<0.05$.

hepatic insulin signaling. A dissection of the hepatic insulin signaling pathway showed that inactivation of GSK3 by AKT led to increased VLDL secretion, suggesting that increased hepatic insulin signaling via IR/AKT with GSK3 inactivation may be an important mechanism leading to increased VLDL lipid and apoB secretion in diabetes and metabolic syndrome.

Despite a deficiency of peripheral and arterial IRs, the $L 1^{B 6} \mathrm{Ldlr} /$ mice had reduced atherosclerosis. Reductions in VLDL and LDL levels of about $50 \%$ in these mice were sufficient to limit atherogenesis, overcoming likely adverse effects of vascular insulin resistance. We recently showed an increase in atherosclerotic lesion area, increased numbers of apoptotic macrophages, and increased necrotic core formation in WTD-fed $L d l r^{-/}$mice transplanted with $L 1^{B 6}$ bone marrow (19). In addition, knockout of the IR in endothelial cells causes an increase in atherosclerosis on a susceptible background (34), and vascular AKT deficiency markedly worsens atherosclerosis, primarily by influencing endothelial NO release and inflammatory gene expression (35). Thus, insulin resistance at the level of endothelial cells or macrophages appears to worsen atherosclerosis, but in $L 1^{B 6} \mathrm{Ldlr}^{-1-}$ mice, this was offset by the reduced VLDL and LDL levels associated with low IR signaling.

We dissected key elements in the hepatic insulin signaling pathway involved in the regulation of VLDL secretion in WT, $\mathrm{Ldlr}^{-1}$, and $o b / o b$ mice and discovered a major role for signaling via AKT and probably GSK3 in the regulation of VLDL secretion. While this appeared to be the dominant pathway responsible for regulation of VLDL secretion, it is important to note that insulin signaling via AKT/mTOR-1 appeared to have the opposite result of signaling via GSK3 (Figure 7C), perhaps due to compensatory feedback loops that are known to increase AKT activity (29). mTOR activity is subject to regulation by nutritional factors (36) and appears to be involved in a mode of regulation of apoB secretion by n-3 long-chain fatty acids involving autophagy (37), and thus it is conceivable that mTOR could have an important direct role in the regulation of VLDL secretion in certain dietary settings. Since AKT inactivates FoxO1 and constitutively active FoxO1 led to an increase in cholesterol levels (38), insulin signaling should suppress VLDL secretion via FoxO1. Indeed, a recent study has shown that FoxO1 directly targets the promoter of MTP, promoting apoB lipidation and VLDL secretion (39). However, MTP mRNA and protein were not changed in $\mathrm{L}^{\mathrm{B}} \mathrm{Ldll}^{-/}$(Figure $3 \mathrm{~B}$ and Supplemental Figure 1C) or ob/ob mice (not shown), and thus changes in MTP expression are probably not involved in our study. In summary, while insulin signaling via AKT/GSK3 had a predominant role in regulation of VLDL secretion in the models we studied, effects on FoxO1 and mTOR could be counterregulatory to AKT/GSK3 signaling and predominate in other settings.

Our conclusions are consistent with other studies showing that insulin induces the key lipogenic transcription factor, SREBP-1c, and its downstream targets in primary hepatocytes $(40,41)$ and 

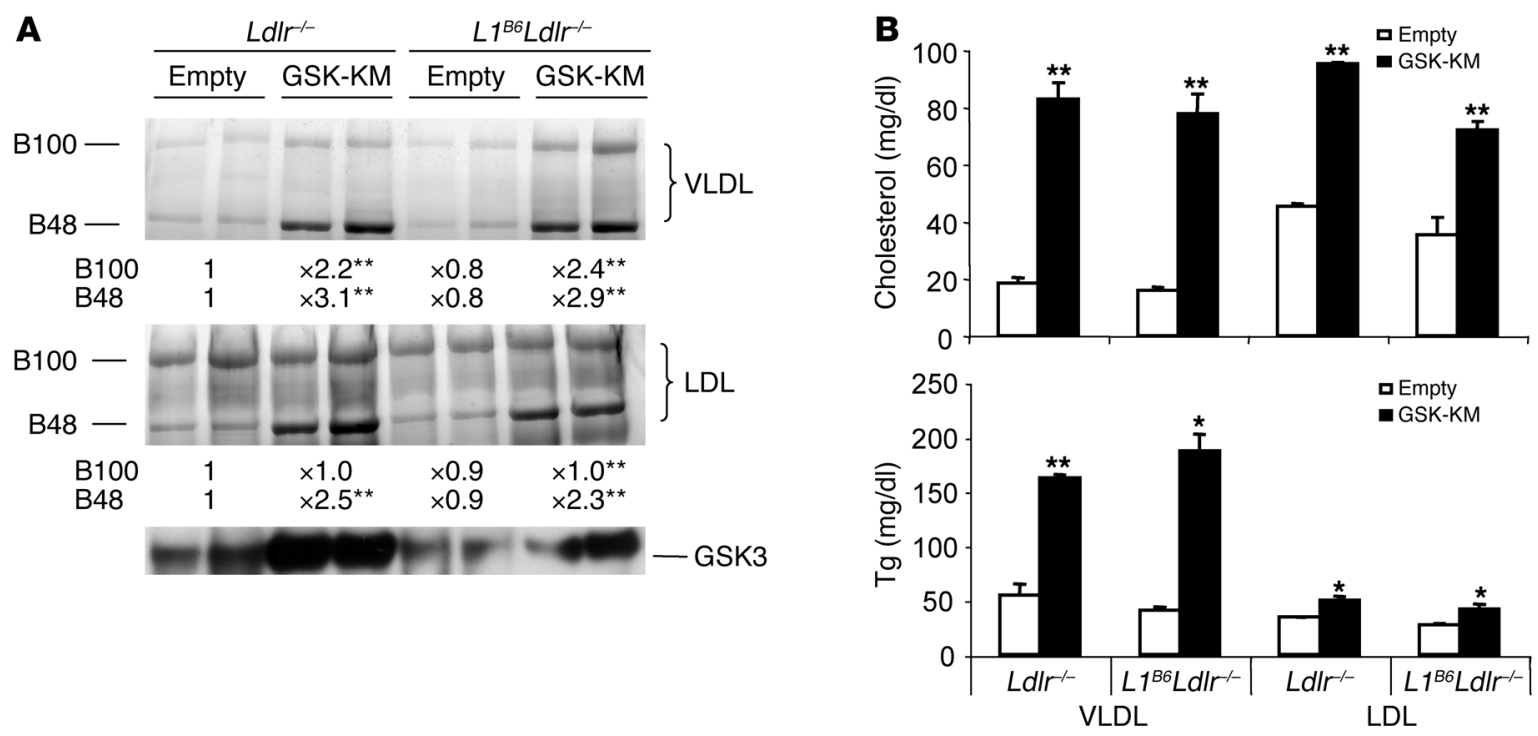

\section{Figure 8}

Effect of AKT downstream effector GSK on plasma apoB levels. (A) Effects of dominant negative GSK (KM) on plasma VLDL and LDL apoB. All $L d l r^{-1}$ and $L 1^{B 6} L_{d l l} r^{--}$mice were 10 to 11 weeks old and fed on chow before virus injection. apoB-containing lipoproteins were quantified and normalized to the amount present in the mice with control empty adenovirus. $n=3 ;{ }^{\star} P<0.05 ;{ }^{* \star} P<0.01$. Lowest panel shows Western blot analysis for hepatic GSK3 expression in infected mice. (B) Plasma lipoprotein cholesterol and Tg levels in $L d l r^{-1-}$ and $L^{B B 6} L d l r^{-1-}$ mice injected with either empty adenovirus (white bars) or GSK-KM (black bars). $n=3$. All data are representative of at least 3 independent experiments.

hepatic overexpression of SREBP leads to increased VLDL Tg and apoB secretion and levels (42). They are also supported by studies showing that AKT overexpression or PTEN knockout leads to increased SREBP expression and increased expression of lipogenic genes in the liver $(43,44)$. A recent cell-culture study has shown that phosphorylation of SREBPs on specific residues (S430 and T426) by GSK3 causes their SCF-mediated ubiquitination and degradation (45). Our studies provide in vivo evidence suggesting a role of GSK3 in regulating VLDL apoB secretion. Nuclear SREBP-1c protein levels were unchanged following myrAKT or dominant negative GSK3 expression in $\mathrm{Ldlr}^{-/}$mice but were moderately decreased in response to constitutively active GSK3 in $o b / o b$ mice (Supplemental Figure 3). Overall, it appears unlikely that GSK3-mediated degradation of SREBP-1c was the major underlying mechanism accounting for decreased VLDL secretion associated with decreased insulin signaling. Other unknown signaling mechanisms not operating via SREBPs appear to be acting downstream of AKT/GSK3 to regulate VLDL apoB and lipid secretion.

The findings in our study in which mice had low hepatic IR levels complement those reported recently using mice with complete liver-specific IR KO (LIRKO) in a background of intact LDL receptors. The finding of reduced expression of SREBP-regulated lipogenic genes and reduced secretion of VLDL lipids is common to the 2 studies. Biddinger et al. (46) reported increased apoB secretion, increased plasma levels of VLDL and LDL, reduced HDL levels, and increased aortic fatty streak formation in LIRKO mice of mixed genetic background fed the Paigen diet. There are several important differences in experimental design that may have contributed to the different outcomes in these studies, such as differences in genetic background and diets. Most importantly, Biddinger et al. used $\mathrm{Ldlr}^{+/+}$mice, while our atherosclerosis studies were carried out in the $\mathrm{Ldlr}^{/-}$strain. The major factor responsible for the atherogenic phenotypes was marked downregulation of hepatic LDL receptor levels and increased VLDL and LDL cholesterol levels on the Paigen diet (46). This is the principal difference and likely the primary reason for the opposite findings concerning VLDL and LDL levels and atherogenesis. This could perhaps also explain the moderate increase in apoB secretion observed by Biddinger et al. (46), since Ldlr is active in the secretory pathway of hepatocytes and thus profound downregulation of hepatic Ldlr levels as observed in the LIRKO mice may lead to increased apoB secretion (47). Another important difference relates to the level of residual hepatic IR expression. Our studies used mice with low levels of IRs in liver ( $10 \%$ of controls in $\mathrm{L1}^{\mathrm{B} 6} \mathrm{Ldlr}{ }^{-/}$mice and $20 \%$ of controls in IR shRNA-treated $L d l r^{-/}$mice) versus absent IRs in the LIRKO mice. The different outcomes of complete and partial knockdown of hepatic IRs could be partly related to developmentally determined structural changes in the liver, including 36\%-50\% decreases in liver weight and mitochondrial and functional defects in LIRKO mice (16), with no comparable changes in mice with up to $95 \%$ IR deficiency including the L1 strain $(48,49)$ and no comparable change in liver weight in $L 1^{B 6} \mathrm{Ldlr}^{-/}$mice $(1.2 \pm 0.07 \mathrm{~g}$ vs. $1.3 \pm 0.08 \mathrm{~g} ; P=0.2)$. It is notable that LIRKO mice had increased hepatic-free and hepatic-esterified cholesterol accumulation on chow or high-cholesterol diets, despite markedly reduced expression of cholesterol biosynthetic genes. This suggests that small, developmentally impaired livers of the LIRKO mice may accumulate dietary cholesterol contributing to downregulation of Ldlrs and cholesterol biosynthetic genes. However, in agreement with the study of Biddinger et al. (46), we found that reducing hepatic insulin signaling via the IR led to reduced levels of Ldlr protein, both in $o b / o b$ mice and WTD-fed WT mice (Figure 10A), though perhaps not as dramatically as observed in the LIRKO mice.

In hyperinsulinemic obese $(o b / o b)$ or lipodystrophic mice, the liver shows paradoxical sensitivity to the actions of insulin on lipogenic gene expression and VLDL secretion but insulin resistance in 
A

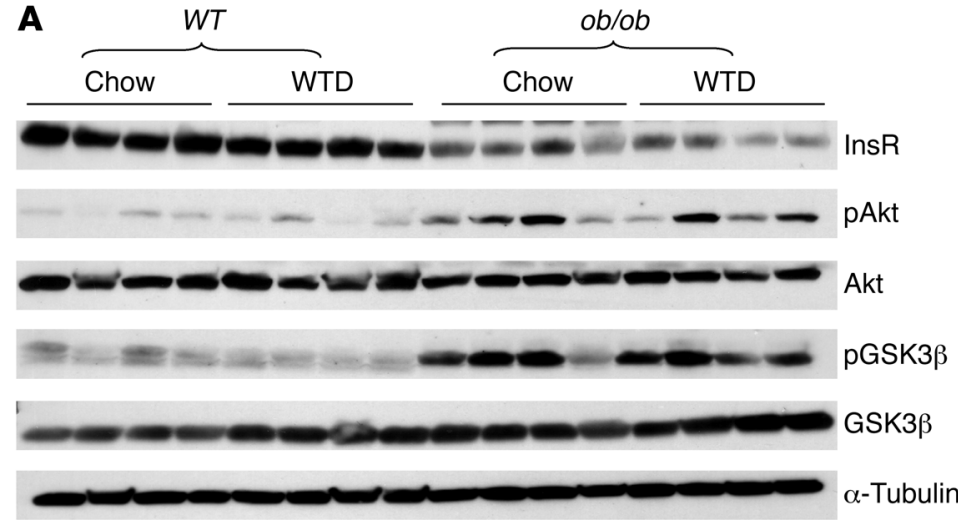

Fold change (ob/wt)

Chow WTD

$\times 0.5^{\star \star} \times 0.4^{\star *}$

$\times 5.9^{*} \times 4.1^{* *}$

$\times 0.9 \times 0.8$

$\times 3.5^{\star} \times 8.5^{\star \star}$

$\times 1.1 \times 1.0$

$\times 0.8 \times 0.8$

\section{Figure 9}

Increased hepatic AKT activity and VLDL and apoB production in ob/ob mice. (A) Western blot analysis of insulin signaling proteins in the livers of WT (C57BL/6J) and 8- to 9-weekold ob/ob male mice fed either chow or WTD for 2 weeks, after a 5-hour fast. Fold changes show the protein expression ratios of ob/ob over WT. $n=4 ;{ }^{*} P<0.05$; ${ }^{\star *} P<0.01$. (B) $\mathrm{Tg}$ production and apoB levels in WT and $o b / o b$ mice. Tg production was determined at indicated times after Triton WR-1339 injection. Plasma from each mouse at 120-minute time point was used for Western blot analysis of apoB. $n=3-4$.

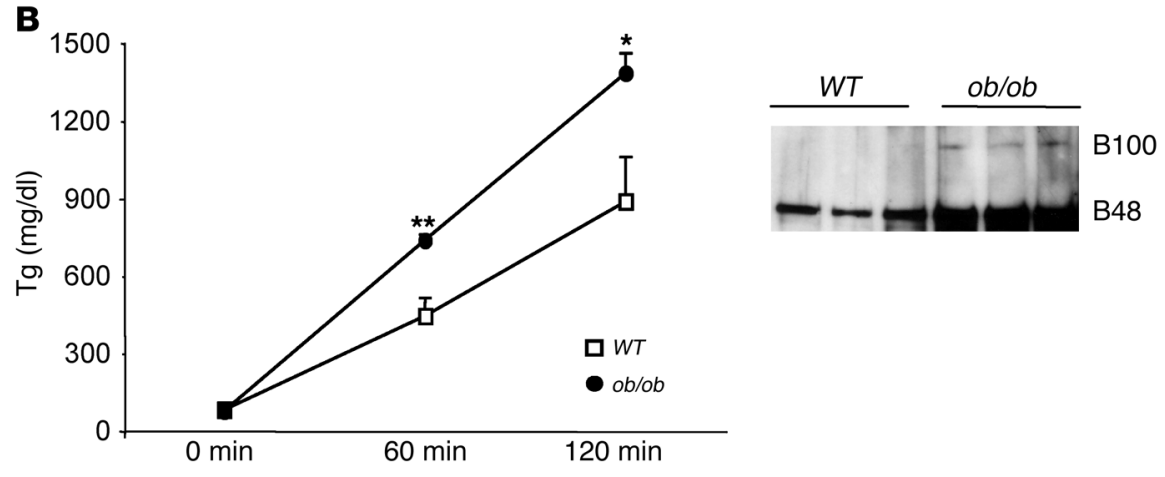

terms of increased expression of genes mediating gluconeogenesis (50). Our mice, $L 1^{B 6} L d l r^{--}$, showed the opposite phenotype - resistance to the lipogenic effects of insulin signaling but sensitivity in terms of gluconeogenic gene expression. Our data suggest that chronically reduced insulin signaling via AKT leads to persistently reduced lipogenic gene expression and VLDL secretion but that there is compensation via an unknown mechanism for the expected increased FoxO1 activity. The divergent control mechanisms for glucose homeostasis and lipogenesis in the liver could be exploited therapeutically in metabolic syndrome or type 2 diabetes, for example, by reducing hepatic gluconeogenesis, hyperinsulinemia, and consequent increased hepatic lipogenesis and VLDL secretion (51). Our findings suggest that a direct partial reduction in hepatic insulin signaling via the IR/AKT/GSK3 axis could reduce VLDL lipid and apoB secretion. However, based on our findings in $o b / o b$ and WT mice (Figure 10A) and in the LIRKO mice as well (46), such a strategy could also reduce hepatic Ldlr levels, without an overall net benefit on plasma VLDL and LDL levels. Interestingly, constitutively active GSK3 seemed to reduce VLDL apoB secretion in $o b / o b$ mice, without reducing Ldlr protein levels (Figure 10F), suggesting divergent downstream effects of insulin signaling on control of Ldlr protein levels and VLDL secretion. Thus, a better understanding of the effects of insulin signaling downstream of AKT could lead to the identification of therapeutic strategies to reduce VLDL secretion, perhaps without adverse effects on gluconeogenesis or hepatic Ldlr expression.

\section{Methods}

Animals. Transgenically rescued $I R^{-/-} \mathrm{L} 1$ strain mice (17) with mixed C57BL/6, FVB, and 129/Sv genetic backgrounds were backcrossed into C57BL/6 mice using marker-aided selection (19). We named this C57BL/6
L1 strain of mice $L 1^{B 6}$. In N7 generation, these $L 1^{B 6}$ mice were further backcrossed into $\mathrm{Ldlr}^{-/}$mice on a C57BL/6 background (002207; The Jackson Laboratory) to produce $\mathrm{L} 1^{B 6} \mathrm{Ldlr} /-$ mice in a uniform C57BL/6 background. The N9 generation male descendants were used for all the experiments performed in this study. Both $\mathrm{L}^{\mathrm{B} 6} \mathrm{Ldl} \mathrm{r}^{-/}$and control $\mathrm{Ldlr}{ }^{-/}$groups contained the same level of human IR transgene (TTR-IR). The mice were genotyped for WT mouse $I R$ allele and human $I R$ transgene by PCR amplification of total genomic DNA extracted from tail biopsies (19). $o b / o b$ and WT C57BL/6 mice were purchased from The Jackson Laboratory. For the atherosclerosis study, all the mice were fed WTD ( $21 \%$ milk fat, $0.15 \%$ cholesterol; TD88137, Harlan Teklad) starting at the age of 8 weeks. The length of WTD feeding is specified in the figure legends. Mice freely accessed food and water and were housed in a pathogen-free facility following animal welfare guidelines. All experiments were reviewed and approved by the Columbia University Institutional Animal Care and Use Committee and were conducted according to that committee's guidelines.

Plasma glucose, insulin, lipid, and hepatic lipid determination. Mice were fasted for 5 hours, and blood samples were collected by retroorbital venous plexus puncture. Plasma was separated by centrifugation and stored at $-70^{\circ} \mathrm{C}$ until analyzed. Total plasma cholesterol, FFA, and HDL cholesterol were measured with Wako enzymatic kits according to the manufacturer's instructions. Tgs, glucose, and insulin were measured with Infinity (Thermo Scientific), Trinder (Sigma-Aldrich), and an ELISA kit (Crystal Chem Inc.), respectively, according to the manufacturers' instructions. For hepatic lipid determination, all the livers were collected after mice were fasted for 5 hours. Hepatic $\mathrm{Tg}$ and cholesterol contents were determined as described (52).

FPLC and ultracentrifugation. $250 \mu$ l of pooled plasma $(n=10)$ from mice fasted for 5 hours was used for FPLC analysis using 2 Sepharose 6 columns in series (Amersham Biosciences). The buffer contained $100 \mathrm{mM}$ Tris and $0.04 \% \mathrm{NaN}_{3}$, $\mathrm{pH} 7.5$; a flow rate of $0.7 \mathrm{ml} / \mathrm{min}$ was used. Cholesterol levels of FPLC fractions were measured using Wako enzymatic kits (Cholesterol E). The major 
A

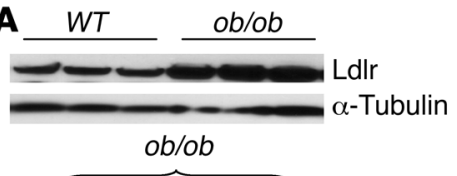

Scrambled IR shRNA

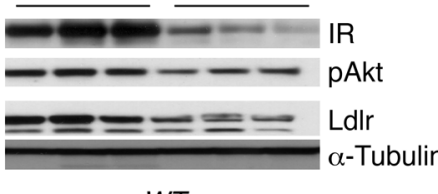

WT

Scrambled IR shRNA

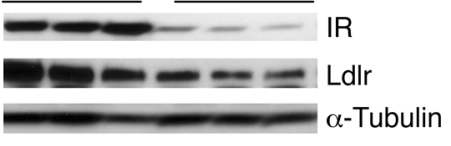

D

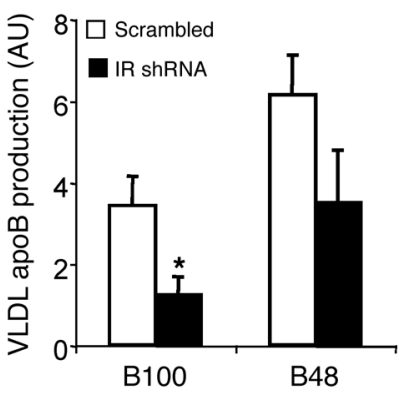

B

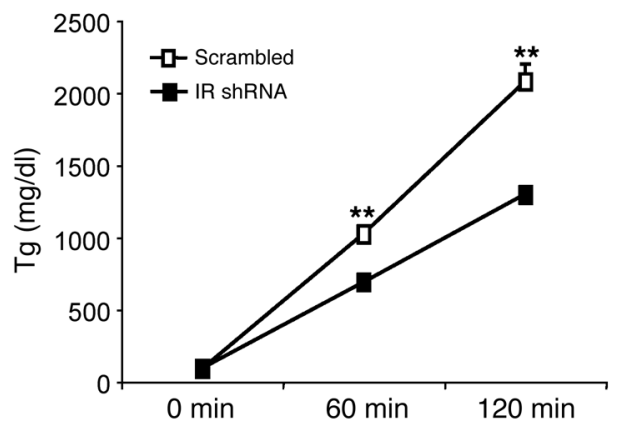

C

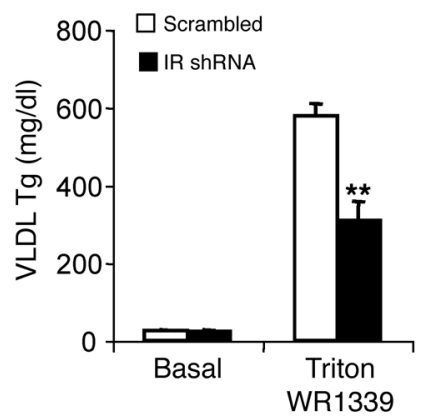

Figure 10

The effect of hepatic IR knockdown and of constitutively active GSK (S9A) on VLDL and apoB production in ob/ob mice. (A) Western blot analysis in upper panel shows Ldlr and $\alpha$-tubulin in WT (C57BL/6J) and ob/ob mice. Center panel shows IR, pAKT, LdIr, and $\alpha$-tubulin in ob/ob mice after injection with scrambled adenovirus or IR shRNA. Lower panel shows IR, Ldlr, and $\alpha$-tubulin from WT mouse treated with scrambled or IR shRNA after 4 weeks of WTD feeding. All livers were collected after a 5-hour fast and 10 days after shRNA adenovirus injection. (B) Tg production in ob/ob mice treated with control adenovirus or IR shRNA. Plasma from each mouse at 0- and 120-minute time points was used for ultracentrifugal preparation of the VLDL fraction. VLDL Tg (C) and VLDL apoB (D) secretion were measured. $n=3-4$; ${ }^{*} P<0.05 ;{ }^{* *} P<0.01$. (E) Effect of constitutively active GSK (S9A) on Tg production and apoB levels in ob/ob mice. After a 5-hour fast, Triton WR-1339 was injected and plasma was collected at indicated times from ob/ob mice injected with either empty adenovirus or GSK-S9A. Plasma from each mouse at the 120-minute time point was used for Western blot analysis of apoB. $n=3-4,{ }^{*} P<0.05,1$-tailed Student's $t$ test. (F) Western blot analysis of Ldlr and $\alpha$-tubulin in ob/ob mice after injection with empty or constitutively active GSK (S9A) adenovirus. All livers were collected after 2 weeks of WTD feeding, followed by a 5-hour fast.

lipoproteins, VLDL $(d<1.006 \mathrm{~g} / \mathrm{ml})$, IDL+LDL $(d=1.006-1.063 \mathrm{~g} / \mathrm{ml})$, and $\operatorname{HDL}(d=1.063-1.21 \mathrm{~g} / \mathrm{ml})$, where $d=$ density, were isolated by sequential density ultracentrifugation of plasma using a TLA-100 rotor (Beckmann Coulter). apoB-containing lipoproteins were run on SDS-PAGE gels and visualized by $0.05 \%$ Coomassie blue staining (Sigma-Aldrich).

Atherosclerosislesion analysis. At 8 weeks of age, mice were switched from regular chow to WTD (21\% milk fat, $0.15 \%$ cholesterol; TD88137, Harlan Teklad) and maintained on this diet for 12 weeks before sacrifice for atherosclerosis studies. Mice were anesthetized using FORANE (Baxter) and killed by cervical dislocation. The aortic roots were dissected and fixed in $10 \%$ formalin (Fisher Scientific) after perfusing hearts with $10 \mathrm{ml}$ of PBS (Invitrogen). Serially paraffin-sectioned proximal aortae were stained with H\&E (Sigma-Aldrich). Lesion areas were quantified using image analysis software Image-Pro Plus 3.0 (MediaCybernetics). Aortic lesion size of each mouse was determined by averaging lesion areas in 5 sections from the same mouse.

apoB and Tg production. The Triton WR-1339 method was used in mice to measure $\mathrm{Tg}$ and $\mathrm{apoB}$ production rate in plasma (53). Mice fasted for five hours were injected with ${ }^{35} \mathrm{~S}$-methionine $(100 \mu \mathrm{Ci}$; GE Healthcare $)$ and Triton WR-1339 (0.5 mg/g body weight; Sigma-Aldrich) simultaneously via tail vein in order to radiolabel apoB and block the clearance of nascent apoBcontaining lipoproteins. Blood samples were collected at $0,30,60$, and 120 minutes after injection. Plasma samples were used for Tg quantification or SDS gel electrophoresis. Gels were dried and exposed to $\mathrm{x}$-ray film to visualize ${ }^{35}$ S-methionine-labeled apoB100 and apoB48 proteins.

Western blot analysis. To examine expression of proteins, liver samples were collected from 5-hour fasted mice or 5 minutes after i.p. injection of insulin (5 U/kg body weight; Sigma-Aldrich) into overnight-fasted mice. Cell lysates were prepared and analysis was performed as described previously (38). Western blot analysis was carried out using the following primary antibodies: anti-AKT (Cell Signaling Technology) anti-P-AKT (Cell Signaling Technology); anti-GSK3 $\beta$ (Cell Signaling Technology); anti-P-GSK3 $\beta$ (Cell Signaling Technology); anti-FoxO1 (Santa Cruz Biotechnology Inc.); anti-P-FoxO1 (Cell Signaling Technology); anti-mTOR (Cell Signaling Technology); antiP-mTOR (Cell Signaling Technology); anti-apoB (Abcam); anti-IR (Santa Cruz Biotechnology Inc.); anti-IRS1 (Upstate; Millipore); anti-IRS2 (Upstate; Millipore); anti-p85 (Upstate; Millipore); anti-Ldlr (Abcam); anti- $\alpha$-tubulin (Abcam); and anti- $\beta$-actin antibody (Sigma-Aldrich). For IRS1-associated p85 and IRS2-associated p85, equal amounts $(\sim 1 \mathrm{mg})$ of liver extracts were 
immunoprecipitated using either anti-IRS1 or anti-IRS2 antibody and precipitant was immunoblotted using anti-p85 antibody. Nuclear extraction from liver was performed using the AY2002 Nuclear Extraction Kit (Panomics), according to the manufacturer's instructions. Antibodies for detecting nuclear SREBP-1c and Lamin A were from Abcam. Protein samples were separated by SDS-PAGE and transferred onto nitrocellulose membranes (BioRad). Blots were probed separately with antibodies as described in respective figures. Following incubation with horseradish peroxidase-conjugated secondary antibodies, proteins were visualized with SuperSignal West Pico Chemiluminescent reagents (Pierce; Thermo Scientific) on $\mathrm{x}$-ray films. The band intensity was quantified using scanning densitometry of the autoradiogram with NIH ImageJ software (http://rsb.info.nih.gov/ij/).

Real-time quantitative PCR analysis. Liver tissues were homogenized and total RNA was isolated using RNAzol B (Tel-Test) according to the manufacturer's instructions. $2 \mu \mathrm{g}$ of total RNA was treated with DNAse I (Ambion; Applied Biosystems) and reverse transcribed at $42^{\circ} \mathrm{C}$ with SuperScript II (Life Technologies). The expression of SREBP-1c and FAS mRNA was measured by real-time quantitative PCR with TaqMan Universal PCR Master Mix (Applied Biosystems). Detection of specific PCR products was performed in triplicate using the $\mathrm{Mx} 4000$ Multiplex Quantitative PCR System (Stratagene), with 1 cycle at $50^{\circ} \mathrm{C}$ for 2 minutes and 1 cycle at $95^{\circ} \mathrm{C}$ for 10 minutes followed by 45 cycles at $95^{\circ} \mathrm{C}$ for 15 seconds and $60^{\circ} \mathrm{C}$ for 1 minute. The expression of ACC, SCD1, acyl-CoA oxidase 1 (Acox1), uncoupling protein 2 (UCP2), SREBP-2, HMG-CoA synthase, HMG-CoA reductase, MTP, apoB, IGFBP1, and PEPCK was measured using SYBR Green PCR core reagents (Applied Biosystems). Quantitative PCR was performed in triplicate using the Mx4000 Multiplex Quantitative PCR System with 1 cycle at $95^{\circ} \mathrm{C}$ for 10 minutes followed by 40 cycles at $90^{\circ} \mathrm{C}$ for 30 seconds, $58^{\circ} \mathrm{C}$ for 30 seconds, and $72^{\circ} \mathrm{C}$ for 1 minute. The relative amounts of specific target amplicons for each primer set were estimated by a standard curve method using Mx4000 software (version 3.01; Stratagene) and were normalized to the copy number of housekeeping gene 36B4. The sequences of PCR probes and primers used are described in Supplemental Table 3.

IR shRNA adenovirus and in vivo study. The sequences against mouse (C57BL/6J) IR mRNA (54) were cloned to BamHI/HindIII sites of DUAL-U6PLAC1 vector, and the recombinant adenoviruses were generated by Vector BioLabs. Prior to in vivo use, both scrambled control adenoviruses and IR shRNA were purified through a cesium chloride gradient. 10- to 11-week-old WTD-fed $L d l r^{-/}$or chow-fed ob/ob mice were injected via tail vein $\left(3 \times 10^{9}\right.$ $\mathrm{PFU} /$ mice) with control adenovirus or IR shRNA. Ten days after injection, the blood and liver tissues were collected from the mice after fasting for 5 hours. Another group of mice receiving the same treatment were used for Triton WR-1339 experiments to examine apoB and $\mathrm{Tg}$ production.
AKT, GSK3, and FoxO1 adenoviruses and in vivo studies. myrAKT and adenoviral vectors expressing constitutively active GSK3 (GSK-S9A, gift from K. Walsh's lab, Boston University School of Medicine, Boston, Massachusetts, USA), dominant negative GSK3 (GSK-KM, gift from K. Walsh's lab), and FoxO1-ADA were previously described (27, $38,55)$. These adenoviruses were purified and concentrated by using AdenoPACK 100 (Sartorius Stedim Biotech) following the manufacturer's instructions. Recombinant empty adenovirus was used as a control (ViraQuest Inc). Virus titer was determined with Adeno-X Rapid Titer Kit (BD Biosciences). myrAKT, GSK-KM, and control adenovirus $\left(5 \times 10^{7}\right.$ $\mathrm{PFU} / \mathrm{g}$ body weight) were injected via tail vein in $\mathrm{L} 1^{B 6} \mathrm{Ldlr^{-- }}$ and control $\mathrm{Ldlr}^{-1-}$ mice. GSK-S9A or control adenovirus $\left(2 \times 10^{7} \mathrm{PFU} / \mathrm{g}\right.$ body weight) was injected via tail vein into $o b / o b$ mice. FoxO1-ADA, at doses of $1,5,10$, and $100 \times 10^{7} \mathrm{PFU} / \mathrm{g}$ body weight, was injected via tail vein into $\mathrm{Ldlr}^{-1-}$ mice. Four days after injection, blood and liver tissues were collected from mice fasted for 5 hours.

In vivo rapamycin and LiCl treatment. 8- to 10-week-old $\mathrm{Ldlr}^{/-}$mice received $400 \mu \mathrm{l}$ of PBS/0.1\% DMSO supplemented with rapamycin $(0.1 \mathrm{mg} / \mathrm{kg}$ or $1 \mathrm{mg} / \mathrm{kg}$ body weight; LC Laboratories) through i.p injection every other day. After the fifth injection, blood and liver tissues were collected from mice fasted for 5 hours. For LiCl treatment, 8- to 10-week-old $\mathrm{Ldlr}^{\circ}$ - mice fed a chow diet received saline with or without $\mathrm{LiCl}$ ( $4 \mathrm{mmol} / \mathrm{kg}$ body weight; SigmaAldrich) through i.p injection every day for 7 days. After 7 days of treatment, blood and liver tissues were collected from mice fasted for 5 hours.

Statistics. Results were expressed as mean \pm SEM ( $n$ is indicated in the figure legends or figures). All statistical data analysis was performed by unpaired 2-tailed Student's $t$ test unless otherwise indicated in the figure legends. $P<0.05$ was considered statistically significant.

\section{Acknowledgments}

We are thankful to Kadisha Collins-Fletcher for technical assistance for lesion quantification and members of the Tall laboratory for helpful discussions. M. Westerterp is supported by the Netherlands Organization for Scientific Research (NWO Rubicon grant 825.07.018). This work was supported by NIH grant HL087123.

Received for publication June 17, 2008, and accepted in revised form January 14, 2009.

Address correspondence to: Seongah Han, Division of Molecular Medicine, Department of Medicine, Columbia University, 630 West 168th St., New York, New York 10032, USA. Phone: (212) 305-4899; Fax: (212) 305-5052; E-mail: sh2068@columbia.edu.
1. Nigro, J., Osman, N., Dart, A.M., and Little, P.J. 2006. Insulin resistance and atherosclerosis. Endocr. Rev. 27:242-259.

2. Reaven, G.M. 2002. Multiple CHD risk factors in type 2 diabetes: beyond hyperglycaemia. Diabetes Obes. Metab. 4(Suppl. 1):S13-S18.

3. Adiels, M., et al. 2005. Overproduction of VLDL1 driven by hyperglycemia is a dominant feature of diabetic dyslipidemia. Arterioscler. Thromb. Vasc. Biol. 25:1697-1703.

4. Myerson, M., et al. 2005. Treatment with high-dose simvastatin reduces secretion of apolipoprotein Blipoproteins in patients with diabetic dyslipidemia. J. Lipid Res. 46:2735-2744.

5. Boden, G., and Shulman, G.I. 2002. Free fatty acids in obesity and type 2 diabetes: defining their role in the development of insulin resistance and beta-cell dysfunction. Eur. J. Clin. Invest. 32(Suppl. 3):14-23.

6. Ginsberg, H.N. 2000. Insulin resistance and cardiovascular disease. J. Clin. Invest. 106:453-458.

7. Sakata, N., Wu, X., Dixon, J.L., and Ginsberg, H.N.
1993. Proteolysis and lipid-facilitated translocation are distinct but competitive processes that regulate secretion of apolipoprotein B in Hep G2 cells. J. Biol. Chem. 268:22967-22970.

8. Ginsberg, H.N. 2002. New perspectives on atherogenesis: role of abnormal triglyceride-rich lipoprotein metabolism. Circulation. 106:2137-2142.

9. Salhanick, A.I., Schwartz, S.I., and Amatruda, J.M. 1991. Insulin inhibits apolipoprotein B secretion in isolated human hepatocytes. Metabolism. 40:275-279.

10. Sparks, J.D., and Sparks, C.E. 1994. Insulin regulation of triacylglycerol-rich lipoprotein synthesis and secretion. Biochim. Biophys. Acta. 1215:9-32.

11. Taghibiglou, C., et al. 2002. Hepatic very low density lipoprotein-ApoB overproduction is associated with attenuated hepatic insulin signaling and overexpression of protein-tyrosine phosphatase $1 \mathrm{~B}$ in a fructose-fed hamster model of insulin resistance. J. Biol. Chem. 277:793-803.

12. Lewis, G.F., and Steiner, G. 1996. Acute effects of insulin in the control of VLDL production in humans. Implications for the insulin-resistant state. Diabetes Care. 19:390-393.

13. Julius, U. 2003. Influence of plasma free fatty acids on lipoprotein synthesis and diabetic dyslipidemia. Exp. Clin. Endocrinol. Diabetes. 111:246-250.

14. Okamoto, H., and Accili, D. 2003. In vivo mutagenesis of the insulin receptor. J. Biol. Chem. 278:28359-28362.

15. Kido, Y., et al. 2000. Tissue-specific insulin resistance in mice with mutations in the insulin receptor, IRS-1, and IRS-2. J. Clin. Invest. 105:199-205.

16. Michael, M.D., et al. 2000. Loss of insulin signaling in hepatocytes leads to severe insulin resistance and progressive hepatic dysfunction. Mol. Cell. 6:87-97.

17. Okamoto, H., Nakae, J., Kitamura, T., Park, B.C., Dragatsis, I., and Accili, D. 2004. Transgenic rescue of insulin receptor-deficient mice. J. Clin. Invest. 114:214-223.

18. Okamoto, H., Obici, S., Accili, D., and Rossetti, L. 2005. Restoration of liver insulin signaling in Insr 
knockout mice fails to normalize hepatic insulin action. J. Clin. Invest. 115:1314-1322.

19. Han, S., et al. 2006. Macrophage insulin receptor deficiency increases ER stress-induced apoptosis and necrotic core formation in advanced atherosclerotic lesions. Cell Metab. 3:257-266.

20. Siri, P., et al. 2001. Post-transcriptional stimulation of the assembly and secretion of triglyceride-rich apolipoprotein B lipoproteins in a mouse with selective deficiency of brown adipose tissue, obesity, and insulin resistance. J. Biol. Chem. 276:46064-46072

21. Lin, H.V., et al. 2007. Adiponectin resistance exacerbates insulin resistance in insulin receptor transgenic knockout mice. Diabetes. 56:1969-1976.

22. Allen, N.D., Norris, M.L., and Surani, M.A. 1990. Epigenetic control of transgene expression and imprinting by genotype-specific modifiers. Cell. 61:853-861.

23. Kido, Y., Philippe, N., Schaffer, A.A., and Accili, D. 2000. Genetic modifiers of the insulin resistance phenotype in mice. Diabetes. 49:589-596.

24. Kako, Y., et al. 1999. Streptozotocin-induced diabetes in human apolipoprotein B transgenic mice. Effects on lipoproteins and atherosclerosis. J. Lipid Res. 40:2185-2194.

25. Nakae, J., Barr, V., and Accili, D. 2000. Differential regulation of gene expression by insulin and IGF-1 receptors correlates with phosphorylation of a single amino acid residue in the forkhead transcription factor FKHR. EMBO J. 19:989-996.

26. Brunet, A., et al. 2001. Protein kinase SGK mediates survival signals by phosphorylating the forkhead transcription factor FKHRL1 (FOXO3a). Mol. Cell. Biol. 21:952-965.

27. Nakae,J., Kitamura, T., Silver, D.L., and Accili, D. 2001. The forkhead transcription factor Foxo1 (Fkhr) confers insulin sensitivity onto glucose-6-phosphatase expression. J. Clin. Invest. 108:1359-1367.

28. Manning, B.D., and Cantley, L.C. 2007. AKT/ PKB signaling: navigating downstream. Cell. 129:1261-1274.

29. Shaw, R.J. 2008. Raptor swoops in on metabolism. Cell Metab. 8:343-344.

30. Beaulieu, J.M., et al. 2008. A beta-arrestin 2 signaling complex mediates lithium action on behavior.
Cell. 132:125-136.

31. Rao, R., et al. 2007. Glycogen synthase kinase 3 inhibition improves insulin-stimulated glucose metabolism but not hypertension in high-fat-fed C57BL/6J mice. Diabetologia. 50:452-460.

32. Rao, R., et al. 2005. Lithium treatment inhibits renal GSK-3 activity and promotes cyclooxygenase 2-dependent polyuria. Am. J. Physiol. Renal Physiol. 288:F642-F649.

33. Gruen, M.L., et al. 2006. Plasma insulin levels predict atherosclerotic lesion burden in obese hyperlipidemic mice. Atherosclerosis. 186:54-64.

34. Rask-Madsen, C., et al. 2007. Loss of insulin signaling in vascular endothelium accelerates atherosclerosis in apolipoprotein $\mathrm{E}$ knockout mice [abstract 0211-OR]. Presented at the 67th American Diabetes Association Scientific Sessions. June 22-26. Chicago, Illinois, USA.

35. Fernandez-Hernando, C., et al. 2007. Loss of akt1 leads to severe atherosclerosis and occlusive coronary artery disease. Cell Metab. 6:446-457.

36. Fisher, T.L., and White, M.F. 2004. Signaling pathways: the benefits of good communication. Curr. Biol. 14:R1005-R1007.

37. Pan, M., et al. 2008. Presecretory oxidation, aggregation, and autophagic destruction of apoproteinB: a pathway for late-stage quality control. Proc. Natl. Acad. Sci. U. S. A. 105:5862-5867.

38. Matsumoto, M., Han, S., Kitamura, T., and Accili, D. 2006. Dual role of transcription factor FoxO1 in controlling hepatic insulin sensitivity and lipid metabolism. J. Clin. Invest. 116:2464-2472.

39. Kamagate, A., et al. 2008. FoxO1 mediates insulindependent regulation of hepatic VLDL production in mice. J. Clin. Invest. 118:2347-2364.

40. Avramoglu, R.K., Basciano, H., and Adeli, K. 2006 Lipid and lipoprotein dysregulation in insulin resistant states. Clin. Chim. Acta. 368:1-19.

41. Shimano, H. 2000. Sterol regulatory element-binding protein-1 as a dominant transcription factor for gene regulation of lipogenic enzymes in the liver. Trends Cardiovasc. Med. 10:275-278.

42. Horton, J.D., Shimano, H., Hamilton, R.L., Brown, M.S., and Goldstein, J.L. 1999. Disruption of LDL receptor gene in transgenic SREBP-1a mice unmasks hyperlipidemia resulting from production of lipid-rich VLDL. J. Clin. Invest. 103:1067-1076.

43. Horie, Y., et al. 2004. Hepatocyte-specific Pten deficiency results in steatohepatitis and hepatocellular carcinomas. J. Clin. Invest. 113:1774-1783.

44. Ono, H., et al. 2003. Hepatic Akt activation induces marked hypoglycemia, hepatomegaly, and hypertriglyceridemia with sterol regulatory element binding protein involvement. Diabetes. 52:2905-2913.

45. Sundqvist, A., et al. 2005. Control of lipid metabolism by phosphorylation-dependent degradation of the SREBP family of transcription factors by SCF(Fbw7). Cell Metab. 1:379-391.

46. Biddinger, S.B., et al. 2008. Hepatic insulin resistance is sufficient to produce dyslipidemia and susceptibility to atherosclerosis. Cell Metab. 7:125-134.

47. Twisk, J., et al. 2000. The role of the LDL receptor in apolipoprotein B secretion. J. Clin. Invest. 105:521-532.

48. Buettner, C., et al. 2005. Severe impairment in liver insulin signaling fails to alter hepatic insulin action in conscious mice. J. Clin. Invest. 115:1306-1313.

49. Kitamura, T., et al. 2004. Mosaic analysis of insulin receptor function. J. Clin. Invest. 113:209-219.

50. Shimomura, I., et al. 2000. Decreased IRS-2 and increased SREBP-1c lead to mixed insulin resistance and sensitivity in livers of lipodystrophic and ob/ob mice. Mol. Cell. 6:77-86.

51. Brown, M.S., and Goldstein, J.L. 2008. Selective versus total insulin resistance: a pathogenic paradox. Cell Metab. 7:95-96.

52. Yvan-Charvet, L., Even, P., Lamande, N., Ferre, P., and Quignard-Boulange, A. 2006. Prevention of adipose tissue depletion during food deprivation in angiotensin type 2 receptor-deficient mice. Endocrinology. 147:5078-5086.

53. Jiang, X.C., et al. 2001. Apolipoprotein B secretion and atherosclerosis are decreased in mice with phospholipid-transfer protein deficiency. Nat. Med. 7:847-852.

54. Seibler, J., et al. 2007. Reversible gene knockdown in mice using a tight, inducible shRNA expression system. Nucleic Acids Res. 35:e54.

55. Kim, H.S., et al. 2002. Regulation of angiogenesis by glycogen synthase kinase-3beta. J. Biol. Chem. 277:41888-41896. 